\title{
Crossover of conductance and local density of states in a single-channel disordered quantum wire
}

\author{
S. Ryu \\ Department of Applied Physics, University of Tokyo, 7-3-1 Hongo Bunkyo-ku, Tokyo 113-8656, Japan \\ C. Mudry \\ Paul Scherrer Institute, CH-5232 Viligen PSI, Switzerland
}

A. Furusaki

Condensed-Matter Theory Laboratory, RIKEN, Wako, Saitama 351-0198, Japan

(Received 8 February 2004; published 18 November 2004)

\begin{abstract}
The probability distribution of the mesoscopic local density of states (LDOS) for a single-channel disordered quantum wire with chiral symmetry is computed in two different geometries. An approximate ansatz is proposed to describe the crossover of the probability distributions for the conductance and LDOS between the chiral and standard symmetry classes of a single-channel disordered quantum wire. The accuracy of this ansatz is discussed by comparison with a large-deviation ansatz introduced by H. Schomerus and M. Titov in Phys. Rev. B 67, 100201 (2003).
\end{abstract}

DOI: $10.1103 /$ PhysRevB.70.195329

PACS number(s): 71.30.+h, 72.15.Rn, 64.60.Fr, 05.40.-a

\section{INTRODUCTION}

The metal-insulator transition induced by disorder in the problem of Anderson localization remains poorly understood from a theoretical point of view. On the one hand, it is true that the prediction for the existence of a metal-insulator transition induced by disorder has been verified by numerical simulations. On the other hand, the critical exponents at the metal-insulating transition measured numerically still cannot be extracted from the field theories which are believed to embody the metal-insulator transition. ${ }^{1}$ The paradigm of this unpleasant situation is the plateau transition in the lowest Landau level of the integer quantum Hall effect. ${ }^{2}$ The development of reliable methods to compute analytically critical properties at a disorder-induced metal-insulator transition remains to this date an open problem.

The simplest known example of disorder-induced criticality in the context of Anderson localization was solved by Dyson in $1953 .{ }^{3}$ According to Dyson, the global density of states (DOS) in the thermodynamic limit and close to the band center is anomalous when a single quantum particle hops with a random amplitude between the nearest-neighbor sites of a chain excluding any other form of disorder, in short, the one-dimensional random hopping problem. Subsequent works showed that Dyson's spectral anomaly is related to a diverging localization length, ${ }^{4-6}$ an anomalous decay of the envelope of wave functions, ${ }^{7}$ and an anomalous probability distribution of the resistance upon approaching the band center. $^{8}$

The coalescence of the concept of universality imported from the theory of critical phenomena with the symmetry classification imported from random matrix theory has led to a classification of diffusive regimes and disorder-induced metal-insulator transitions in terms of 10 universality classes that are uniquely characterized by the intrinsic symmetries preserved by the disorder (assumed weak) for any given dimensionality of space. ${ }^{9}$ According to this classification, Dyson's singular DOS is a trademark of the chiral universality classes. The chiral universality classes are realized in problems of Anderson localization for which the Hamiltonian anticommutes with some unitary operator for all realizations of the disorder and in which case the DOS is known to be singular at the band center in zero, ${ }^{10,11}$ quasi-one, ${ }^{12-14}$ and two dimensions. ${ }^{15-18}$ Even though the chiral universality classes are critical at the band center, they have been far less studied than the orthogonal, unitary, and symplectic universality classes, which we will refer to as the standard universality classes. For example, the probability distribution of the so-called mesoscopic local density of states (LDOS) has been known since 1989 from the work of Altshuler and Prigodin for a single chain with weak on-site disorder, ${ }^{19-21}$ whereas it has not yet been computed for the onedimensional random-hopping problem. The first aim of this paper is to fill this gap.

If a diffusive regime exists, perturbative techniques can be used to describe the crossovers between universality classes. ${ }^{22,23}$ However, little is known quantitatively on the crossover between two different universality classes, here defined in terms of nonlinear-sigma models, say, from the diffusive to the localized regimes. This is even true in one dimension, which is by far the most studied laboratory for Anderson localization, as was recently illustrated by a flurry of works on the validity of one-parameter scaling in one dimension. ${ }^{24-28}$ The absence of a diffusive regime renders the concept of universality classes ambiguous in one dimension in that symmetry alone does not specify a universality class in one dimension. For example, the ratio of the localization length to the mean-free path in a wire of finite width interpolates smoothly between its two limiting values for fully preserved or completely broken time-reversal symmetry, respectivley, as a function of a weak magnetic field. ${ }^{29}$ Similarly, fine tuning of microscopic parameters is required to achieve delocalization (i.e., diverging localization length) of quasiparticles at the fermi energy in a dirty superconducting wire of finite thickness with both broken spin-rotation and time-reversal symmetries whereas delocalization be- 
comes generic in the thick-wire scaling limit. ${ }^{23,30}$ One can nevertheless define a symmetry class in one dimension by demanding that single-parameter scaling holds. Lack of universality in one dimension can then be understood as the fact that there is no scaling limit for which single-parameter scaling becomes a generic property of an ensemble of random microscopic Hamiltonians of a given symmetry. Symmetry classes in one dimension cannot be construed as an enumeration of stable or unstable fixed points of some putative effective field theory. Computing the crossover between symmetry classes in one dimension nevertheless remains a welldefined problem.

Time-reversal symmetry cannot be broken for a spinless particle constrained to move in a one-dimensional and simply connected world, in which case the only possible crossover between symmetry classes takes place between the chiral and standard classes. The second aim of this paper is to compute the crossover between these two symmetry classes in one dimension for the probability distributions of the conductance and of the LDOS from the ballistic to the localized regimes assuming a weak disorder at the microscopic level. In that regard, our results are complementary to the analysis made by Schomerus and Titov of the validity of oneparameter scaling in one dimension in which they computed exactly the first four cumulants of the logarithm of the conductance to leading order in the ratio of the length of the chain to the localization length assuming a weak disorder at the microscopic level. ${ }^{25,26} \mathrm{We}$ thus propose an approximate ansatz for the crossover whereby the approximation consists of assuming that the phase and amplitude of the reflection coefficient separate. By comparing the cumulants of the Lyapunov exponent of the transfer matrix computed with our approximate ansatz to the ones computed with the largedeviation ansatz of Schomerus and Titov deep in the localization regime, we deduce that our ansatz captures the first cumulant, but fails with the higher cumulants.

The paper is organized as follows. We formulate the onedimensional problem of Anderson localization as a continuous Schrödinger equation with a relativistic kinetic energy in Sec. II. A functional renormalization group equation is derived for the probability distribution of the reflection coefficient in Sec. III. Exact solutions to these coupled functional renormalization group equations are given for the chiral and the standard classes, as well as an approximate solution that describes the crossovers between these two classes in Sec. IV. The accuracy of the approximate solution for the crossover regime is estimated in Sec. V. The probability distribution of the LDOS is computed exactly in the chiral and standard classes whereas it is computed approximately in the crossover regime between these two classes in Sec. VI. We conclude in Sec. VII.

\section{MODEL}

In this paper we want to investigate the statistical properties of the LDOS $\nu$ and dimensionless conductance $g$ for a single quantum particle, which is (i) restricted to a simply connected and strictly one-dimensional (1D) geometry and (ii) subjected to a static and weak random environment (dis- (a)

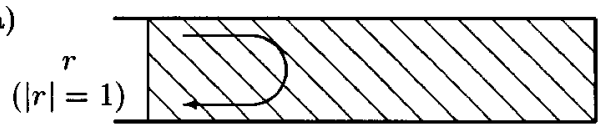

(b)

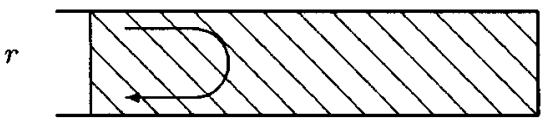

(c)

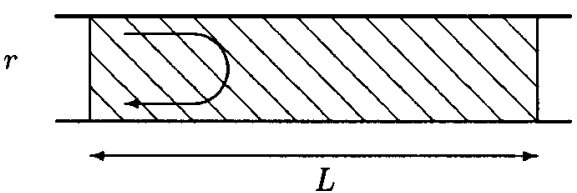

FIG. 1. In this paper, we will consider two different simply connected geometries, which are imposed by suitable boundary conditions at $y=-L / 2$ and $y=+L / 2$, respectively, for a strictly 1D disordered quantum wire. In geometry (a) and (b) the disordered quantum wire is closed to the right and open to the left where it is connected to an ideal lead. The particle in geometry (b) is subjected to absorption whereas it is not in geometry (a). In geometry (c) the disordered quantum wire is open to the left and to the right where it is connected to ideal leads.

order) that interpolates smoothly between the standard and chiral symmetry classes. Condition (i) implies that timereversal symmetry is preserved for every realization of the disorder. In 1D and assuming a metallic ground state in the absence of the disorder, the ground state consists of two distinct Fermi points. Plane waves at the Fermi points are called left- and right-movers, respectively. In the standard symmetry class, the channels for forward and backward scatterings induced by an on-site disorder potential in the basis of leftand right-movers are equally likely up to nonrandom oscillatory factors. In the chiral symmetry class, the disorder potential is off-diagonal in the basis of left- and right-movers. Presuming weak disorder in condition (ii) allows us to choose a kinetic energy that is a first-order differential operator, for it only makes sense to linearize the spectrum about the two Fermi points when the disorder potential is weak. We thus choose to model the dynamics of the single quantum particle and the static random environment by the Hamiltonian

$$
H:=-\sigma_{3} i \frac{d}{d y}-\sum_{\mu=0}^{2} \sigma_{\mu} v_{\mu}(y),
$$

where we have set the Fermi velocity $v_{\mathrm{F}}$ and $\hbar$ to one. The boundary conditions that will be imposed in the sequel are depicted in Fig. 1. They all obey condition (i). We have denoted by $\sigma_{0}$ the unit $2 \times 2$ matrix and by $\sigma_{1,2,3}$ the $2 \times 2$ Pauli matrices. The static random environment is represented by three independent potentials $v_{\mu}(y) \in \mathbb{R}, \mu=0,1,2$ that we choose, for mathematical convenience, to be supported on the interval $-L / 2 \leqslant y \leqslant+L / 2$, and to be white-noise and Gaussian distributed with vanishing mean (disorder averaging is denoted by $\langle\ldots\rangle)$

$$
\left\langle v_{\mu}(y)\right\rangle=0,
$$




$$
\left\langle v_{\mu}(y) v_{\nu}\left(y^{\prime}\right)\right\rangle=2 g_{\mu} \delta_{\mu \nu} \delta\left(y-y^{\prime}\right) .
$$

The three variances $g_{0,1,2}$ carry the dimensions of inverse length and need not be equal. A finite value

$$
\left\langle v_{2}(y)\right\rangle=\Delta
$$

can also be easily accommodated in our formalism. ${ }^{31}$ In this case, the clean system is insulating as $\Delta$ opens up a dimerization gap at the Fermi energy and the random environment mimics to a first approximation the static fluctuations of the phonons responsible for the dimerization sufficiently close to the Peierls transition. ${ }^{32}$ The problem of Anderson localization in the continuum defined by Eq. (2.1a), (2.1b), and (2.1c) is a coarse-grained version of a single particle hopping on a simply connected chain with a uniform nearest-neighbor hopping amplitude subjected to weak and independent random fluctuations of an on-site potential and of the nearestneighbor hopping amplitude.

The generic symmetry obeyed by Eq. (2.1a) is timereversal invariance,

$$
\sigma_{1} H^{*} \sigma_{1}=H \text {. }
$$

If the condition

$$
g_{0}=g_{1}=0
$$

is satisfied, or, for that matter, any equivalent condition obtained by a redefinition of the Pauli matrices through a position independent $S U(2)$ rotation around $\sigma_{3}$, Eq. (2.1a) has an additional chiral symmetry in that it anticommutes with $\sigma_{1}$,

$$
\sigma_{1} H \sigma_{1}=-H .
$$

Consequently, all nonvanishing eigenvalues of $H$ come in pairs with opposite signs. The origin of the chiral symmetry of the continuum model for any given realization of the disorder is a sublattice symmetry of an appropriate lattice regularization. A microscopic random Hamiltonian realizing the chiral symmetry is, for example, a tight-binding Hamiltonian on a hypercubic lattice with strictly vanishing on-site energies and random hopping matrix elements restricted to nearest-neighbor sites, in short, the random hopping problem.

The LDOS, DOS, conductance, shot-noise power, etc., can all be extracted from the elements of the $2 \times 2$ scattering matrix $S_{E}$ at the energy $E$ that connects incoming ("i") and outgoing ("o") states

$$
\left(\begin{array}{l}
\psi^{\mathrm{o}, \mathrm{L}} \\
\psi^{\mathrm{o}, \mathrm{R}}
\end{array}\right)_{E}=S_{E}\left(\begin{array}{c}
\psi^{\mathrm{i}, \mathrm{L}} \\
\psi^{\mathrm{i}, \mathrm{R}}
\end{array}\right)_{E} \equiv\left(\begin{array}{ll}
r & t^{\prime} \\
t & r^{\prime}
\end{array}\right)_{E}\left(\begin{array}{c}
\psi^{\mathrm{j}, \mathrm{L}} \\
\psi^{\mathrm{j}, \mathrm{R}}
\end{array}\right)_{E} .
$$

The amplitude for incoming and outgoing waves to the leftand right-hand sides of the disordered region is denoted by $\psi^{\mathrm{i} / \mathrm{o}, \mathrm{L} / \mathrm{R}}$ here. The matrix elements $r, r^{\prime} \in \mathrm{C}$ are the reflection coefficients whereas the matrix elements $t, t^{\prime} \in \mathrm{C}$ are the transmission coefficients. Alternatively, one can work with the $2 \times 2$ transfer matrix $\mathcal{M}_{E}$ at the energy $E$, which follows from the scattering matrix (2.6) through the basis transformation implied by

$$
\left(\begin{array}{c}
\psi^{\mathrm{\rho}, \mathrm{R}} \\
\psi^{\mathrm{i}, \mathrm{R}}
\end{array}\right)_{E}=\mathcal{M}_{E}\left(\begin{array}{c}
\psi^{\mathrm{i}, \mathrm{L}} \\
\psi^{\mathrm{o}, \mathrm{L}}
\end{array}\right)_{E}
$$

Conservation of probability dictates that the scattering matrix is unitary or, equivalently, that the transfer matrix is pseudo unitary

$$
\begin{gathered}
\left(S_{E}\right)^{\dagger} \sigma_{0} S_{E}=S_{E} \sigma_{0}\left(S_{E}\right)^{\dagger}=\sigma_{0}, \\
\left(\mathcal{M}_{E}\right)^{\dagger} \sigma_{3} \mathcal{M}_{E}=\mathcal{M}_{E} \sigma_{3}\left(\mathcal{M}_{E}\right)^{\dagger}=\sigma_{3} .
\end{gathered}
$$

Conservation of probability also implies the existence of the polar decompositions

$$
\begin{gathered}
S_{E}=\left(\begin{array}{cc}
v^{\prime *} & 0 \\
0 & u
\end{array}\right)_{E}\left(\begin{array}{cc}
-\tanh x & \operatorname{sech} x \\
\operatorname{sech} x & \tanh x
\end{array}\right)_{E}\left(\begin{array}{cc}
v & 0 \\
0 & u^{\prime *}
\end{array}\right)_{E}, \\
\mathcal{M}_{E}=\left(\begin{array}{cc}
u & 0 \\
0 & u^{\prime}
\end{array}\right)_{E}\left(\begin{array}{cc}
\cosh x & \sinh x \\
\sinh x & \cosh x
\end{array}\right)_{E}\left(\begin{array}{cc}
v & 0 \\
0 & v^{\prime}
\end{array}\right)_{E},
\end{gathered}
$$

where $u_{E}, u_{E}^{\prime}, v_{E}, v_{E}^{\prime}$ are independent complex numbers with $\left|u_{E}\right|^{2}=\left|u_{E}^{\prime}\right|^{2}=\left|v_{E}\right|^{2}=\left|v_{E}^{\prime}\right|^{2}=1$ and $x_{E} \in \mathbb{R}$. The polar decomposition is not unique. For example, the sign of $x_{E}$ can be absorbed into a redefinition of $u_{E}, u_{E}^{\prime}, v_{E}, v_{E}^{\prime}$. The variable $x_{E}$, when restricted to the half line $[0, \infty[$, has the geometrical interpretation of a radial coordinate on a Riemannian manifold. ${ }^{33,34}$ The time-reversal symmetry of the Hamiltonian implies the transformation laws

$$
\begin{gathered}
S_{E}=\left(S_{E}\right)^{T}, \\
\sigma_{1} \mathcal{M}_{E}^{*} \sigma_{1}=\mathcal{M}_{E},
\end{gathered}
$$

which enforce the constraints $u_{E}^{\prime}=u_{E}^{*}$ and $v_{E}^{\prime}=v_{E}^{*}$ or, equivalently, $t_{E}=t_{E}^{\prime}$. The chiral symmetry of the Hamiltonian implies the transformation laws

$$
\begin{gathered}
S_{+E}=\left(S_{-E}\right)^{\dagger}, \\
\sigma_{1} \mathcal{M}_{+E} \sigma_{1}=\mathcal{M}_{-E},
\end{gathered}
$$

which enforce the constraints $u_{+E}^{\prime}=u_{-E}$ and $v_{+E}^{\prime}=v_{-E}$ or, equivalently, $r_{+E}=r_{-E}^{*}, r_{+E}^{\prime}=r_{-E}^{\prime *}, t_{+E}=t_{-E}^{\prime *}$. Then, at the band center $E=0$ with the condition (2.4), the scattering matrix becomes Hermitian.

Under the assumptions that (i) the disorder is weak and (ii) $u_{E}, u_{E}^{\prime}, v_{E}, v_{E}^{\prime}$ are all independently and uniformly distributed on the unit circle in the complex plane, it was essentially shown in Ref.35 that the probability distribution $\mathcal{X}$ of $x_{E} \geqslant 0$ obeys the Fokker-Planck equation

$$
\frac{\partial \mathcal{X}(x ; L)}{\partial L}=\frac{1}{4 \ell} \frac{\partial}{\partial x} \sinh (2 x) \frac{\partial}{\partial x} \operatorname{csch}(2 x) \mathcal{X}(x ; L)
$$

in the geometry of Fig. 1(c). The mean-free path $\ell$ is some function of $g_{0}, g_{1}, g_{2}$, and $E$. In a more general context of multi channel quantum wires, assumption (ii) is known as the isotropy assumption, ${ }^{36}$ whereas the Fokker-Planck equation (2.12) is a special case of the Dorokhov-Mello-Pichard- 
Kumar (DMPK) equation. ${ }^{37,36} \mathrm{~A}$ consequence of assumption (ii) is one-parameter scaling as encoded by the FokkerPlanck equation (2.12), i.e., the probability distribution of $x$ and, consequently, of $g=\operatorname{sech}^{2} x$ depends on the single dimensionless parameter $L / \ell$. It is evident that assumption (ii) breaks down at the band center $E=0$ and with the chiral condition (2.4) because $u_{E=0}=u_{E=0}^{\prime}$ and $v_{E=0}=v_{E=0}^{\prime}$ are then both real valued. Correspondingly, the Fokker-Planck equation, obeyed by the probability distribution $\mathcal{X}$ of $-\infty<x_{E=0}<+\infty$, is different from Eq. (2.12) and given by the diffusion equation

$$
\frac{\partial \mathcal{X}(x ; L)}{\partial L}=\frac{1}{2 \ell} \frac{\partial^{2} \mathcal{X}(x ; L)}{\partial x^{2}}
$$

in the geometry of Fig. 1(c). ${ }^{8,38,31}$ The mean-free path $\ell$ is some function of $g_{2}$. Again, the Fokker-Planck equation (2.13) encodes one-parameter scaling as the probability distribution of $x$ and, consequently, of $g=\operatorname{sech}^{2} x$ depends on the single dimensionless parameter $L / \ell$. There are no known multiple-parameter scaling equations for the LDOS, DOS, conductance, etc., which describe the crossover between the two limiting cases described by Eqs. (2.12) and (2.13) for values of $L$ ranging from the ballistic ( $L$ smaller than the mean-free path) to the localized regime ( $L$ larger than the mean-free path) to the best of our knowledge (see Refs. 39-41 for discussions of two-parameter scaling). We fill this gap in the remainder of the paper.

\section{FUNCTIONAL RENORMALIZATION GROUP EQUATIONS}

We shall show in Secs. IV and VI that the statistical properties of the dimensionless conductance $g$ and of the LDOS $\nu$ follow from the knowledge of the statistical properties of the reflection coefficient

$$
r=: \sqrt{R} \exp (i \phi)
$$

which has been decomposed into its square modulus $0 \leqslant R$ $\leqslant 1$ and phase $0 \leqslant \phi<2 \pi$. For a disordered wire of length $L+\delta L$ as depicted in Fig. 2, the reflection coefficient $r_{L+\delta L}$ is related to the entries of the scattering matrix $S_{\delta L}$ for the slice of length $\delta L$ and to the entry $r_{L}$ of the scattering matrix $S_{L}$ for the much longer segment of length $L$ by the composition law

$$
r_{L+\delta L}=r_{\delta L}+t_{\delta L}^{\prime}\left(1-r_{L} r_{\delta L}^{\prime}\right)^{-1} r_{L} t_{\delta L}
$$

When the width $\delta L$ of the slice is much larger than the lattice spacing $a$, but much smaller than the mean-free path $\ell$, here defined by 22

$$
\left\langle r_{\delta L} r_{\delta L}^{*}\right\rangle=: \frac{\delta L}{\ell}
$$

we infer the continuous Langevin process

$$
\frac{d R}{d L}=-4 \mathcal{E}^{\prime \prime} R+2 \sqrt{R}(1-R)\left(v_{1} \sin \phi-v_{2} \cos \phi\right),
$$

(a)

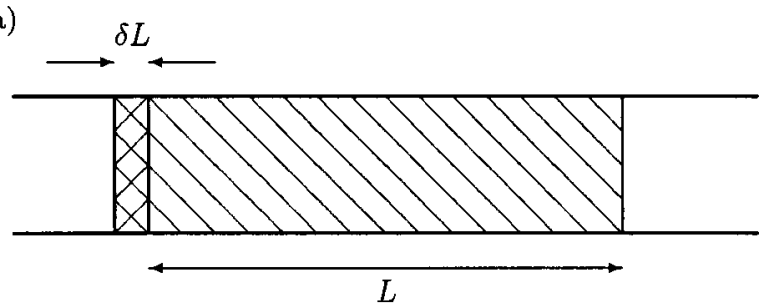

(b)

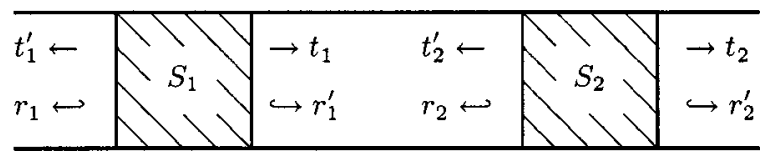

FIG. 2. (a) A thin slice of length $\delta L$ with $a \ll \delta L \ll \ell \ll L$ is added to the left of the disordered region of length $L$. (b) Two disordered regions 1 and 2 with scattering matrices $S_{1}$ and $S_{2}$, respectively, in a quantum wire.

$$
\frac{d \phi}{d L}=2\left(\mathcal{E}^{\prime}+v_{0}\right)+\left(v_{1} \cos \phi+v_{2} \sin \phi\right)\left(\sqrt{R}+\frac{1}{\sqrt{R}}\right) \text {, }
$$

by using the relations

$$
\begin{aligned}
& r_{\delta L}=\left(i v_{1}-v_{2}\right) \delta L, \quad t_{\delta L}=1+i\left(v_{0}+E\right) \delta L, \\
& r_{\delta L}^{\prime}=\left(i v_{1}+v_{2}\right) \delta L, \quad t_{\delta L}^{\prime}=1+i\left(v_{0}+E\right) \delta L,
\end{aligned}
$$

which are valid up to first order in the disorder potentials $v_{0,1,2}$, and by expanding the right-hand side of Eq. (3.2) to the same order. We note in passing that the mean-free path (3.3) is simply given by

$$
\ell=\frac{1}{2\left(g_{1}+g_{2}\right)}
$$

in the Born approximation. For later convenience, we have continued the energy $E$ to the upper part of the complex plane, $E=\mathcal{E}^{\prime}+i \mathcal{E}^{\prime \prime}, \mathcal{E}^{\prime \prime} \geqslant 0$. The continuous Langevin process (3.4) can also be formulated as the Fokker-Planck equation obeyed by the joint probability distribution function $P(R, \phi ; L)$. Using the standard methods of Chapter 3 in Ref. 42 say, one finds ${ }^{43}$

$$
\begin{aligned}
\frac{\partial P}{\partial t}= & F_{0} P-F_{R} \frac{\partial P}{\partial R}-F_{\phi} \frac{\partial P}{\partial \phi}+\frac{1}{2} G_{R R} \frac{\partial^{2} P}{\partial R^{2}}+G_{R \phi} \frac{\partial^{2} P}{\partial R \partial \phi} \\
& +\frac{1}{2} G_{\phi \phi} \frac{\partial^{2} P}{\partial \phi^{2}},
\end{aligned}
$$

where

$$
\begin{gathered}
F_{0}(R, \phi)=2 \omega+2(2 R-1)-6 \zeta R \cos 2 \phi, \\
F_{R}(R, \phi)=-2 \omega R-(R-1)(5 R-1)+6 \zeta R(R-1) \cos 2 \phi, \\
F_{\phi}(R, \phi)=+\varepsilon+\frac{\zeta}{2}\left(5 R+4+\frac{1}{R}\right) \sin 2 \phi,
\end{gathered}
$$




$$
\begin{gathered}
G_{R R}(R, \phi)=2 R(1-R)^{2}-2 \zeta R(1-R)^{2} \cos 2 \phi, \\
G_{R \phi}(R, \phi)=\zeta\left(1-R^{2}\right) \sin 2 \phi, \\
G_{\phi \phi}(R, \phi)=4 \zeta_{0}+\frac{1}{2}\left(R+2+\frac{1}{R}\right)+\frac{\zeta}{2}\left(R+2+\frac{1}{R}\right) \cos 2 \phi .
\end{gathered}
$$

We have introduced the short-hand notations

$$
\begin{aligned}
& g_{ \pm}:=g_{1} \pm g_{2}, \quad t:=L / \ell, \\
& \varepsilon:=\mathcal{E}^{\prime} / g_{+}, \quad \omega:=\mathcal{E}^{\prime \prime} / g_{+}, \\
& \zeta:=g_{-} / g_{+}, \quad \zeta_{0}:=g_{0} / g_{+} .
\end{aligned}
$$

Observe that the dependence on the polar angle $\phi$ arises solely from $\cos 2 \phi$ and $\sin 2 \phi$ in the Fokker-Planck equation (3.7). Thus, provided initial and boundary conditions are also periodic on the interval $[0, \pi[$, the joint probability distribution $P(R, \phi ; L)$ is periodic on the interval $[0, \pi[$ even though the phase $\phi$ is originally defined modulo $2 \pi$. The periodicity $\pi$ is a consequence of the Langevin process (3.4) being invariant under $\sqrt{R} \rightarrow-\sqrt{R}$ and $\phi \rightarrow \phi+\pi$. A dimerization gap $\left\langle v_{2}(y)\right\rangle=\Delta$ produces the changes

$$
\begin{gathered}
F_{0}(R, \phi) \rightarrow F_{0}(R, \phi)+\frac{\Delta}{g_{+}}(R-1) \frac{1}{\sqrt{R}} \cos \phi, \\
F_{R}(R, \phi) \rightarrow F_{R}(R, \phi)+\frac{\Delta}{g_{+}}(R-1) \sqrt{R} \cos \phi, \\
F_{\phi}(R, \phi) \rightarrow F_{\phi}(R, \phi)+\frac{\Delta}{2 g_{+}}(R+1) \frac{1}{\sqrt{R}} \sin \phi .
\end{gathered}
$$

The polar decomposition (2.9) suggests the change of variable

$$
\sqrt{R}=\tanh |x|,
$$

in terms of which the Langevin process (3.4) becomes

$$
\begin{gathered}
\frac{d x}{d L}=-\mathcal{E}^{\prime \prime} \sinh 2 x+v_{1} \sin \phi-v_{2} \cos \phi, \\
\frac{d \phi}{d L}=2\left(\mathcal{E}^{\prime}+v_{0}\right)+\frac{2}{\tanh 2 x}\left(v_{1} \cos \phi+v_{2} \sin \phi\right) .
\end{gathered}
$$

In turn, the joint probability distribution function $W(x, \phi ; L)$ obeys the Fokker-Planck equation

$$
\begin{aligned}
\frac{\partial W}{\partial t}= & J_{0} W-J_{x} \frac{\partial W}{\partial x}-J_{\phi} \frac{\partial W}{\partial \phi}+\frac{1}{2} K_{x x} \frac{\partial^{2} W}{\partial x^{2}}+K_{x \phi} \frac{\partial^{2} W}{\partial x \partial \phi} \\
& +\frac{1}{2} K_{\phi \phi} \frac{\partial^{2} W}{\partial \phi^{2}},
\end{aligned}
$$

where

$$
\begin{aligned}
J_{0}(x, \phi)= & 2 \omega \cosh 2 x+\frac{1}{\sinh ^{2} 2 x} \\
& -\zeta\left(\frac{1}{\sinh ^{2} 2 x}+\frac{2}{\tanh ^{2} 2 x}\right) \cos 2 \phi,
\end{aligned}
$$

$$
J_{x}(x, \phi)=-\omega \sinh 2 x+\frac{1}{2} \frac{1}{\tanh 2 x}-\frac{3}{2} \frac{\zeta}{\tanh 2 x} \cos 2 \phi,
$$

$$
J_{\phi}(x, \phi)=\varepsilon+\zeta\left(\frac{1}{\sinh ^{2} 2 x}+\frac{3}{\tanh ^{2} 2 x}\right) \sin 2 \phi,
$$

and

$$
\begin{gathered}
K_{x x}(x, \phi)=\frac{1}{2}(1-\zeta \cos 2 \phi), \\
K_{x \phi}(x, \phi)=\frac{\zeta}{\tanh 2 x} \sin 2 \phi, \\
K_{\phi \phi}(x, \phi)=4 \zeta_{0}+\frac{2}{\tanh ^{2} 2 x}+\zeta \frac{2}{\tanh ^{2} 2 x} \cos 2 \phi .
\end{gathered}
$$

The Langevin process (3.11) or the Fokker-Planck equation (3.12) are invariant under $x \rightarrow-x$ and $\phi \rightarrow \phi+\pi$.

Equations (3.7) and (3.12) are, from a conceptual point of view, the main result of this paper as they determine under what conditions one-parameter scaling holds in a 1D weakly disordered quantum wire. For example, Eqs. (3.12) encodes the functional renormalization group flow of the joint probability distribution of the radial coordinate $|x|=\operatorname{arctanh} \sqrt{R}$ and phase $\phi$ of the reflection coefficient $r$. If it is the property that the functional renormalization group flow of the probability distribution of $x$ depends solely on the dimensionless ratio $L / \ell$ aside from $x$, which is understood as oneparameter scaling for $x,{ }^{40}$ then two examples of oneparameter scaling can be constructed from Eqs. (3.12) as follows. First, the Fokker-Planck (DMPK) Eq. (2.12) follows from insertion of the ansatz

$$
W(x, \phi ; L)=\frac{1}{2 \pi} \mathcal{X}(x ; L)
$$

into Eqs. (3.12) with $\omega=0$ and from integration over $\phi$ $\in[0,2 \pi[$ of the resulting equation. Second, the FokkerPlanck (DMPK) equation (2.13) follows from insertion of the ansatz

$$
W(x, \phi ; L)=\frac{1}{2}[\delta(\phi-0)+\delta(\phi-\pi)] \mathcal{X}(x ; L)
$$

with $g_{0}=g_{1}=\omega=\varepsilon=0$ into Eqs. (3.12) and from integration over $\phi$ of the resulting equation. One-parameter scaling is not the rule for generic initial and boundary conditions of Eqs. (3.12) and for generic values of $\varepsilon$ and $g_{0,1,2}$ due to the absence of a diffusive regime in $1 \mathrm{D} .^{23}$ This is most clearly seen by the fact that the localization length $\xi$, as defined by 


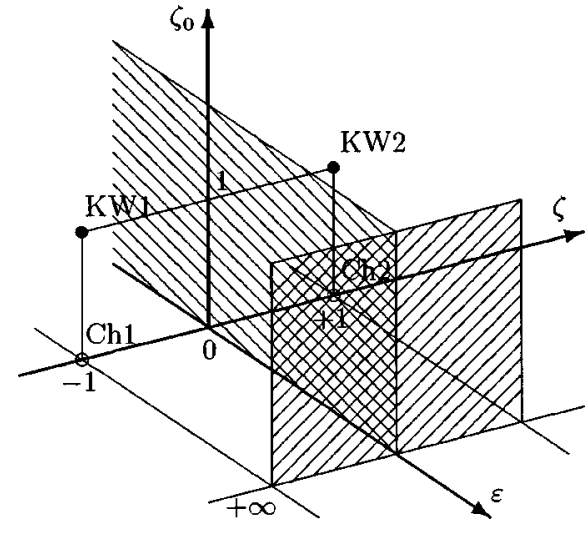

FIG. 3. Parameter space for the Fokker-Planck equations (3.7) or (3.12). The point $\mathrm{Ch} 1$ has the coordinates $\varepsilon=\zeta_{0}=0, \zeta=-1$. It represents the chiral symmetry class. The point KW 1 has the coordinates $\varepsilon=0, \zeta_{0}=-\zeta=1$. It represents the Kappus-Wegner anomaly. The two shaded planes represent the standard symmetry class. The regions $\zeta>0$ and $\zeta<0$ are equivalent in that they are related by a rotation by an angle $\pi$ around $\sigma_{3}$ of the Pauli matrices in the Hamiltonian (2.1a). Especially, this rotation relates $\mathrm{Ch} 1$ and $\mathrm{KW} 1$ to the points Ch 2 and KW 2, respectively.

the typical dependence of the conductance $g=\operatorname{sech}^{2} x$ on $L$ $\gg \ell$, is finite, $\xi=2 \ell$, for the standard symmetry class, but diverges in the chiral limit $g_{0}=g_{1}=\omega=\varepsilon=0$. Hence, the localization length must depend on a second microscopic parameter aside from $\ell$ for generic values of $\varepsilon$ and $g_{0,1,2}$. Equations (3.7) and (3.12) describe the full crossover from the chiral to the standard class with no restrictions on the values of $x$ and $L$.

For any given absorption $\omega \geqslant 0$, the parameter space $\left(\zeta, \zeta_{0}, \varepsilon\right)$ with $\zeta_{0} \geqslant 0$ and $\zeta, \varepsilon \in \mathrm{R}$ of Eqs. (3.7) and (3.12) is three-dimensional and is depicted in Fig. 3. Remarkably, the parameter $\zeta$ always enters Eqs. (3.7) and (3.12) as a prefactor to $\cos 2 \phi$ or $\sin 2 \phi$ and, conversely, $\cos 2 \phi$ or $\sin 2 \phi$ are always multiplied by $\zeta$. As we shall see in Sec. IV this property turns out to be crucial in our study of the crossover between the chiral and standard symmetry classes.

Equation (3.7) was derived in the standard class by Abrikosov, Melnikov, and Kumar, and by Rammal and Doucot among others starting from the continuous nonrelativistic Schrödinger equation. ${ }^{44-47}$ The large $x$ limit

$$
\begin{aligned}
\frac{\partial W}{\partial t} \approx & \left(\omega e^{2 x}-2 \zeta \cos 2 \phi\right) W+\left(\frac{\omega}{2} e^{2 x}-\frac{1}{2}+\frac{3}{2} \zeta \cos 2 \phi\right) \frac{\partial W}{\partial x} \\
& +(-\varepsilon-3 \zeta \sin 2 \phi) \frac{\partial W}{\partial \phi}+\frac{1}{4}(1-\zeta \cos 2 \phi) \frac{\partial^{2} W}{\partial x^{2}} \\
& +\zeta \sin (2 \phi) \frac{\partial^{2} W}{\partial x \partial \phi}+\left(2 \zeta_{0}+1+\zeta \cos 2 \phi\right) \frac{\partial^{2} W}{\partial \phi^{2}}
\end{aligned}
$$

of Eq. (3.12) was derived by Schomerus and Titov when $\omega$ $=0$ and $g_{0}=g_{1}$ starting from a Langevin process for the eigenfunctions of the discrete Schrödinger equation on a chain. ${ }^{25,26}$

\section{SOLUTIONS TO THE FUNCTIONAL RENORMALIZATION GROUP EQUATIONS}

Exact and approximate solutions to the Fokker-Planck equation (3.7) are derived in this section. We will consider three regimes of parameter in Fig. 3. The first regime corresponds to the chiral symmetry class. The second regime corresponds to the standard symmetry class. The third regime describes a specific path connecting the two symmetry classes.

The chiral symmetry class is given by the condition

$$
\varepsilon=0,
$$

which defines the band center of the energy eigenvalue spectrum of Hamiltonian (2.1a) when

$$
\zeta=-1, \quad \zeta_{0}=0, \quad\left(g_{0}=g_{1}=0\right),
$$

i.e., when Hamiltonian (2.1a) anticommutes with $\sigma_{1}$ as in Eq. (2.5). The standard symmetry class occurs whenever

$$
\zeta=0 \quad\left(g_{1}=g_{2}\right)
$$

or

$$
1 / \zeta_{0}=0
$$

or

$$
1 /|\varepsilon|=0,
$$

since any one of these three conditions decouples $R$ from $\phi$ in Eq. (3.7) and is consistent with a stationary probability distribution of $\phi$, which is uniformly distributed. In this paper we shall only consider the crossover between the chiral and standard symmetry classes along the line

$$
\zeta=-1, \quad \zeta_{0}=0, \quad 0<\varepsilon<\infty .
$$

We must naturally choose the initial and boundary conditions to be imposed on the solutions to the Fokker-Planck equation (3.7). Our choice is motivated by the computation of the probability distribution of the LDOS that we will carry out in Sec. VI. Two geometries depicted in Fig. 6 will be considered in Sec. VI. The relevant geometries for the probability distribution of the reflection coefficient $r$ that enter the computation of the LDOS are depicted in Fig. 1. In Figs. 1(a) and 1(b) the disordered quantum wire is closed on the righthand side and connected to a reservoir on the left-hand side. In Fig. 1(a) conservation of probability holds. In Fig. 1(b) conservation of probability does not hold due to the presence of an absorption encoded by a finite imaginary part $\mathcal{E}^{\prime \prime}$ $=\omega g_{+}$of $E$. In Fig. 1(c) the disordered quantum wire is open at both ends and flux conservation (no absorption) is assumed. We choose the ideal initial condition $r=1$ and $r=0$ when $L=0$ for the semiopen and fully open geometries of Fig. 1, respectively. In the chiral symmetry class, we demand that the phase of $r$ is either 0 or $\pi$ with probability $1 / 2$ initially. In the standard symmetry class, we demand that the phase of $r$ is uniformly distributed in the interval $[0,2 \pi[$ initially. In view of the periodicity of the Fokker-Planck equation (3.7) we always impose initial conditions with a periodicity of $\pi$. 
We shall now construct exact solutions to the FokkerPlanck equation (3.7) in the chiral and standard symmetry classes, respectively, for the three geometries of Fig. 1. We shall also solve the Fokker-Planck equation (3.7) in the crossover regime (4.3) for the geometry of Fig. 1(a). We could not construct exact solutions to the Fokker-Planck equation (3.7) in the crossover between the chiral and standard symmetry classes for the geometries of Figs. 1(b) and 1(c). We shall construct, however, an approximate solution to the Fokker-Planck equation (3.7) in a crossover regime that interpolates between the exact solutions for the geometries of Figs. 1(b) and 1(c). The accuracy of this approximate solution will be discussed in Sec. V.

\section{A. Disordered quantum wire closed on the right-hand side without absorption}

In geometry 1a, an incoming plane wave from an ideal lead is perfectly reflected by the disordered quantum wire. Hence, the reflection coefficient $r$ of the disordered quantum wire must be a pure phase, $r r^{*}=1$, for all $t=L / \ell$. We thus insert the separation-of-variable ansatz

$$
P(R, \phi ; t)=\delta(R-1) \Phi(\phi ; t)
$$

into the Fokker-Planck equation (3.7) that, after integration over $R$, reduces to

$$
\begin{aligned}
\frac{\partial \Phi}{\partial t}= & -2 \zeta \cos (2 \phi) \Phi-[\varepsilon+3 \zeta \sin (2 \phi)] \frac{\partial \Phi}{\partial \phi} \\
& +\left[2 \zeta_{0}+1+\zeta \cos (2 \phi)\right] \frac{\partial^{2} \Phi}{\partial \phi^{2}} .
\end{aligned}
$$

By assumption, we have switched off the absorption $\omega=0$. Our choice of initial condition shall depend on the proximity to the parameter regimes (4.1) and (4.2), but shall always be periodic on the interval $[0, \pi[$.

\section{Chiral symmetry class}

In the chiral symmetry class (4.1), the Fokker-Planck equation $(4.4 \mathrm{~b})$ reduces to

$$
\frac{\partial \Phi}{\partial t}=2 \cos (2 \phi) \Phi+3 \sin (2 \phi) \frac{\partial \Phi}{\partial \phi}+[1-\cos (2 \phi)] \frac{\partial^{2} \Phi}{\partial \phi^{2}}
$$

and has the normalized, stationary, and periodic solution

$$
\Phi(\phi)=\frac{1}{2} \delta(\phi-0)+\frac{1}{2} \delta(\phi-\pi)
$$

whenever the phase $\phi$ is 0 or $\pi$ with probability $1 / 2$ initially. The same conclusion could have been anticipated by inspection of the Langevin process (3.4) or from the fact that the chiral symmetry class (4.1a) and (4.1b) imposes the condition that the scattering matrix is Hermitian, see Eq. (2.11a).

\section{Standard symmetry class}

In the standard symmetry class (4.2a), $\cos 2 \phi$ and $\sin 2 \phi$ drop out from the Fokker-Planck equation (4.4b):

$$
\frac{\partial \Phi}{\partial t}=-\varepsilon \frac{\partial \Phi}{\partial \phi}+\left(2 \zeta_{0}+1\right) \frac{\partial^{2} \Phi}{\partial \phi^{2}} .
$$

Its normalized, stationary, and periodic solution is

$$
\Phi(\phi)=\frac{1}{2 \pi}
$$

whenever the phase $\phi$ of $r$ is uniformly distributed initially. More generally, the solution to Eq. (4.6a) converges to the uniform probability distribution (4.6b) as soon as $t \gg 1$ for any given initial probability distribution. This is also true for the standard symmetry class (4.2b) and (4.2c) as $\cos (2 \phi)$ and $\sin (2 \phi)$ are ineffective in these limits.

When $g_{1} \neq g_{2}$ and $|\varepsilon| \lesssim 1$, the stationary probability distribution of $\phi$ need not be uniform in [0,2 $\pi[$. For example, consider the case $\zeta=+1, \varepsilon=0, \zeta_{0}=\frac{1}{2}(1+\zeta)=1$, for which the Fokker-Planck equation (4.4b) reduces to

$$
\frac{\partial \Phi}{\partial t}=-2 \cos (2 \phi) \Phi-3 \sin (2 \phi) \frac{\partial \Phi}{\partial \phi}+[3+\cos (2 \phi)] \frac{\partial^{2} \Phi}{\partial \phi^{2}}
$$

and has the stationary and normalized solution ${ }^{25,48}$

$$
\Phi(\phi)=\frac{\sqrt{2 \pi}}{\Gamma^{2}(1 / 4) \sqrt{1+\cos ^{2} \phi}}
$$

with a periodicity of $\pi$. Here $\Gamma(z)$ denotes the value of the gamma function for $z \in \mathrm{C}$. Equation (4.7b) is a special case of the stationary solution

$$
\Phi(\phi) \propto \frac{1}{\sqrt{2 \zeta_{0}+1+\zeta \cos (2 \phi)}}
$$

to Eq. (4.4b) with $\varepsilon=0$ as shown by Titov. ${ }^{49}$ In the chiral limit $\zeta \rightarrow-1$ and $\zeta_{0} \rightarrow 0$, this solution becomes unnormalizable and we have to use Eq. (4.5b) as a stationary solution instead.

\section{Crossover regime}

For simplicity, we consider the crossover regime (4.3)..$^{50}$ The chiral symmetry class (4.1) is reached when $\varepsilon=0$. The standard symmetry class (4.2c) is reached when $|\varepsilon| \gg 1$. In the crossover regime, the Fokker-Planck equation (4.4b) reduces to

$$
\frac{\partial \Phi}{\partial t}=-\varepsilon \frac{\partial \Phi}{\partial \phi}+2 \frac{\partial}{\partial \phi}\left(\sin \phi \frac{\partial}{\partial \phi} \sin \phi\right) \Phi .
$$

A stationary solution to Eq. (4.8) is constructed from the solution

$$
\Phi(\phi)=\frac{\mathcal{N}}{\sin \phi} \int_{\phi}^{\pi} d \phi^{\prime} \frac{e^{+(\varepsilon / 2)\left(\cot \phi^{\prime}-\cot \phi\right)}}{\sin \phi^{\prime}},
$$

valid for $\varepsilon>0$ and on the interval $[0, \pi[$, by periodic extension to the interval $[0,2 \pi[$. The constant $\mathcal{N}$ is chosen so that the solution (4.9) is properly normalized. The solution interpolates between Eqs. (4.5b) and (4.6b) as $\varepsilon \rightarrow 0$ and $\varepsilon \rightarrow \infty$, respectively, as it should be. Equation (4.9) is an important 
intermediary result as we will use it to construct an approximation to the probability distributions for the conductance and LDOS in the crossover regime that interpolate between the exact chiral and standard limiting probability distributions.

\section{B. Disordered quantum wire closed on the right-hand side with absorption}

In geometry $1 \mathrm{~b}$, an incoming plane wave from an ideal lead is not perfectly reflected by the disordered quantum wire due to a finite absorption $\omega=\mathcal{E}^{\prime \prime} / g_{+}$. Hence, the reflection coefficient $r$ of the disordered quantum wire is not a pure phase anymore, $r=\sqrt{R} \exp (i \phi)$.

\section{Chiral symmetry class}

In the chiral symmetry class (4.1a) and (4.1b), the phase $\phi$ and squared magnitude $R$ of the reflection coefficient $r$ must necessarily separate, since $0 \leqslant r \leqslant 1$ is necessarily real valued,

$$
P(R, \phi ; t)=\left[\frac{1}{2} \delta(\phi-0)+\frac{1}{2} \delta(\phi-\pi)\right] \mathcal{R}(R ; t) .
$$

After insertion of Eq. (4.10) into the Fokker-Planck equation (3.7) one finds that $\mathcal{R}(R ; t)$ obeys

$$
\begin{aligned}
\frac{\partial R}{\partial t}= & {[2 \omega+2(3 R-2)] \mathcal{R}+[2 \omega R+3(1-R)(1-3 R)] \frac{\partial \mathcal{R}}{\partial R} } \\
& +2 R(1-R)^{2} \frac{\partial^{2} \mathcal{R}}{\partial R^{2}}
\end{aligned}
$$

with the stationary solution

$$
R(R)=\mathcal{N} \frac{\exp [-\omega /(1-R)]}{\sqrt{R}(1-R)} .
$$

The constant $\mathcal{N}$ is chosen so that the solution (4.11b) is properly normalized (this is always possible for $\omega>0$ ). The stationary solution $(4.11 \mathrm{~b})$ has a square-root singularity at $R$ $=0$, an essential singularity at $R=1$, and is normalizable for $R \in[0,1]$. We shall see below that the probability for $R$ to be in the vicinity of 0 is enhanced in the chiral symmetry class (4.1) compared to the standard symmetry class (4.2).

\section{Standard symmetry class}

In the standard symmetry class (4.2), the phase $\phi$ and squared magnitude $R$ of the reflection coefficient $r$ can reasonably be taken to separate in view of the initial condition of a uniformly distributed phase $\phi$,

$$
P(R, \phi ; t)=\frac{1}{2 \pi} \mathcal{R}(R ; t) .
$$

After insertion of the separation-of-variable ansatz (4.12) into the Fokker-Planck equation (3.7) one finds that $\mathcal{R}(R ; t)$ obeys

$$
\begin{aligned}
\frac{\partial \mathcal{R}}{\partial t}= & {[2 \omega+2(2 R-1)] \mathcal{R}+[2 \omega R+(1-R)(1-5 R)] \frac{\partial \mathcal{R}}{\partial R} } \\
& +R(1-R)^{2} \frac{\partial^{2} \mathcal{R}}{\partial R^{2}}
\end{aligned}
$$

with the stationary and normalized solution

$$
\mathcal{R}(R)=2 \omega e^{2 \omega} \frac{\exp [-2 \omega /(1-R)]}{(1-R)^{2}} .
$$

The stationary solution (4.13b) has an essential singularity when $R=1$. The stationary solution (4.13b) was found by Pradhan and Kumar. ${ }^{51}$ When the initial condition on the probability distribution of $\phi$ is not that of a uniform distribution, it is presumed that the deviations of the solution to the Fokker-Planck equation (3.7) from the separation-ofvariable ansatz (4.12) with the stationary solution (4.13b) are short transients. We do not know of an analytical verification of this assumption.

\section{Crossover regime}

There is no compelling reason to believe that the squared amplitude $R$ and the phase $\phi$ of the reflection coefficient $r$ separate in the crossover regime. However, short of an explicit exact solution to the Fokker-Planck equation (3.7) in the crossover regime, we shall nevertheless pursue a strategy relying on an approximation built on a separation-of-variable ansatz that allows us to interpolate between the chiral and standard symmetry classes. One possible alternative to the separation-of-variable ansatz that we tried is a perturbative expansion about the standard symmetry class (4.2) using a Fourier expansion of the joint probability distribution for $R$ and $\phi$. However, this approach has the drawback that it cannot be expected to interpolate all the way to the chiral symmetry class (4.1) in view of the nature of the singularities characterizing the limiting probability distributions. For this reason we will not present the perturbative approach in this paper.

Our starting point is the separation-of-variable ansatz

$$
P(R, \phi ; t)=\mathcal{R}(R ; t) \Phi(\phi ; t) .
$$

We further assume that the probability distribution function for the phase $\phi$ shows a quick relaxation to its stationary solution, i.e., $\Phi(\phi ; t)$ is assumed to be $t$-independent,

$$
\Phi(\phi ; t)=\Phi(\phi) .
$$

Insertion of Eq. (4.14) into the Fokker-Planck equation (3.7) followed by an integration over $\phi$ yields

$$
\begin{aligned}
\frac{\partial \mathcal{R}}{\partial t}= & {[2 \omega+2(2 R-1)-2 \zeta \alpha(R-1)] \mathcal{R} } \\
& +[2 \omega R+(1-R)(1-5 R)-2 \zeta \alpha(1-R)(1-2 R)] \frac{\partial \mathcal{R}}{\partial R} \\
& +(1-\zeta \alpha) R(1-R)^{2} \frac{\partial^{2} \mathcal{R}}{\partial R^{2}},
\end{aligned}
$$


where the real constant $\alpha$ is the ratio of two Fourier expansion coefficients,

$$
\alpha:=\frac{\int_{0}^{2 \pi} d \phi \cos (2 \phi) \Phi(\phi)}{\int_{0}^{2 \pi} d \phi \Phi(\phi)} .
$$

A stationary solution to Eq. (4.15a) is

$$
\mathcal{R}(R)=\mathcal{N} \frac{\exp \{-2 \omega /[(1-\zeta \alpha)(1-R)]\}}{R^{-\zeta \alpha /(1-\zeta \alpha)}(1-R)^{2 /(1-\zeta \alpha)}} .
$$

This solution reduces to the known results in the standard [Eq. (4.13b)] and chiral [Eq. (4.11b)] symmetry classes. In the standard symmetry class (4.2) the probability distribution of the phase is uniform $\Phi(\phi)=1 / 2 \pi$ and hence $\zeta \alpha=0$, whereas in the chiral symmetry class (4.1) it is a sum of the two delta functions $\Phi(\phi)=\frac{1}{2} \delta(\phi-0)+\frac{1}{2} \delta(\phi-\pi), \alpha=1, \zeta=$ -1 . There remains considerable arbitrariness in the choice of $\Phi$ since many different $\Phi$ share the same $\alpha$. In practice we will choose the stationary solution (4.9) because we then recover the true solution in the semiopen geometry of Fig. 1 (a) as $\omega \rightarrow 0$. We postpone to Sec. V the discussion of the accuracy of the approximation implied by the separation-ofvariable ansatz (4.15a) with the choice (4.9) for the stationary probability distribution of $\phi$.

\section{Disordered quantum wire opened at both ends without absorption}

In geometry $1 \mathrm{c}$, an incoming plane wave from an ideal lead is partially reflected and partially transmitted by the disordered quantum wire. Following Abrikosov ${ }^{44}$ we trade the squared magnitude $R$ of the reflection coefficient $r$ for the resistance (inverse of conductance)

$$
\varrho:=\frac{1}{1-R} \in[1, \infty[
$$

under which

$$
Q(\varrho, \phi ; t)=P(R, \phi ; t) \frac{d R}{d \varrho}
$$

obeys the Fokker-Planck equation

$$
\begin{aligned}
\frac{\partial Q}{\partial t}= & A_{0} Q-A_{\varrho} \frac{\partial Q}{\partial \varrho}-A_{\phi} \frac{\partial Q}{\partial \phi}+\frac{1}{2} B_{\varrho \varrho} \frac{\partial^{2} Q}{\partial \varrho^{2}}+B_{\varrho \phi} \frac{\partial^{2} Q}{\partial \varrho \partial \phi} \\
& +\frac{1}{2} B_{\phi \phi} \frac{\partial^{2} Q}{\partial \phi^{2}},
\end{aligned}
$$

where

$$
\begin{gathered}
A_{0}(\varrho, \phi)=2 \omega(2 \varrho-1), \\
A_{\varrho}(\varrho, \phi)=-2 \omega \varrho(\varrho-1)-(2 \varrho-1), \\
A_{\phi}(\varrho, \phi)=\varepsilon+\frac{\zeta}{2} \frac{2 \varrho^{2}-2 \varrho+1}{\varrho(\varrho-1)} \sin 2 \phi,
\end{gathered}
$$

and

$$
\begin{gathered}
B_{\varrho \varrho}(\varrho, \phi)=2 \varrho(\varrho-1)(1-\zeta \cos 2 \phi), \\
B_{\varrho \phi}(\varrho, \phi)=\zeta(2 \varrho-1) \sin 2 \phi, \\
B_{\phi \phi}(\varrho, \phi)=4 \zeta_{0}+\frac{1}{2} \frac{(2 \varrho-1)^{2}}{\varrho(\varrho-1)}+\frac{\zeta}{2} \frac{(2 \varrho-1)^{2}}{\varrho(\varrho-1)} \cos 2 \phi .
\end{gathered}
$$

\section{Chiral symmetry class}

In the chiral symmetry class (4.1) insertion of the separation-of-variable ansatz

$$
Q(\varrho, \phi ; t)=\left[\frac{1}{2} \delta(\phi-0)+\frac{1}{2} \delta(\phi-\pi)\right] \mathcal{Q}(\varrho ; t)
$$

into the Fokker-Planck equation (4.17) yields

$$
\frac{\partial \mathcal{Q}}{\partial t}=2 \mathcal{Q}+3(2 \varrho-1) \frac{\partial \mathcal{Q}}{\partial \varrho}+2 \varrho(\varrho-1) \frac{\partial^{2} \mathcal{Q}}{\partial \varrho^{2}}
$$

with the initial condition

$$
\mathcal{Q}(\varrho ; t=0)=\delta(\varrho-1) .
$$

The normalized solution to Eq. (4.19) has the integral representation

$$
\begin{aligned}
\mathcal{Q}(\varrho ; t)= & \frac{1}{\pi} \frac{\varrho^{-1 / 2}}{\sqrt{4 \pi t^{\prime 3}}} \int_{0}^{1} d u \frac{1}{(1-u) \sqrt{\varrho-u}} \\
& \times \int_{0}^{1} d v \frac{D(u, v ; \varrho)}{\sqrt{v(1-v)}} e^{-D^{2}(u, v ; \varrho) / 4 t^{\prime}}
\end{aligned}
$$

with the auxiliary function and variable

$$
D(u, v ; \varrho):=\ln \left[\frac{\varrho-u}{v u(1-u)}\right], \quad t^{\prime}:=2 t
$$

The integrations over $u$ and $v$ can be performed in closed form,

$$
\mathcal{Q}(\varrho ; t)=\frac{1}{\sqrt{2 \pi t \varrho(\varrho-1)}} \exp \left[-\frac{\left(\operatorname{arccosh} \varrho^{1 / 2}\right)^{2}}{2 t}\right] .
$$

By shifting the minimum of the resistance $\varrho$ from 1 to 0 , $\rho:=\varrho-1$, we recover the same asymptotics for the probability distribution of $\rho$ computed by Stone and Joanopoulos ${ }^{8}$ with a log-normal distribution of the nearest-neighbor hopping amplitudes of the discrete Schrödinger equation when $\rho \rightarrow 0$ or $\rho \rightarrow \infty$. The change of variable $g=1 / \varrho$ yields the probability distribution

$$
\mathcal{G}(g ; t)=\frac{1}{\sqrt{2 \pi t(1-g)} g} \exp \left[-\frac{\left(\operatorname{arccosh} g^{-1 / 2}\right)^{2}}{2 t}\right]
$$

for the conductance $g$ in the chiral symmetry class (4.1). The same result follows from solving the diffusion (DMPK) equation (2.13) and performing the change of variables $g$ $=1 / \cosh ^{2} x .^{22}$ 


\section{Standard symmetry class}

In the standard symmetry class (4.2) insertion of the separation-of-variable ansatz

$$
Q(\varrho, \phi ; t)=\frac{1}{2 \pi} \mathcal{Q}(\varrho ; t)
$$

into the Fokker-Planck equation (4.17) yields

$$
\frac{\partial \mathcal{Q}}{\partial t}=(2 \varrho-1) \frac{\partial \mathcal{Q}}{\partial \varrho}+\varrho(\varrho-1) \frac{\partial^{2} \mathcal{Q}}{\partial \varrho^{2}}
$$

with the initial condition

$$
\mathcal{Q}(\varrho ; t=0)=\delta(\varrho-1) .
$$

As shown by Abrikosov, ${ }^{44}$ the normalized solution to Eq. (4.23a) and (4.23b) has the integral representation

$$
\begin{aligned}
\mathcal{Q}(\varrho ; t) & =\frac{e^{-t / 4}}{\sqrt{4 \pi t^{3}}} \int_{0}^{1} d u \frac{e^{-\left[D_{0}(u ; \varrho)\right]^{2} / 4 t}}{[u(1-u)(\varrho-u)]^{1 / 2}} D_{0}(u ; \varrho) \\
& =\sqrt{\frac{4}{\pi t^{3}}} \int_{y_{\varrho}}^{+\infty} d y \frac{y e^{-(t / 4)-\left(y^{2} / t\right)}}{\left(\cosh ^{2} y-\varrho\right)^{1 / 2}}
\end{aligned}
$$

with $y_{\varrho}:=-\frac{1}{2} \ln \left[2 \varrho\left(1-\sqrt{1-\varrho^{-1}}\right)-1\right]$ and

$$
D_{0}(u ; \varrho)=\ln \left[\frac{\varrho-u}{u(1-u)}\right] \text {. }
$$

The change of variable $g=1 / \varrho$ yields the probability distribution

$$
\mathcal{G}(g ; t)=\sqrt{\frac{4}{\pi t^{3} g^{3}}} \int_{y_{g}}^{+\infty} d y \frac{y e^{-(t / 4)-\left(y^{2} / t\right)}}{\left(g \cosh ^{2} y-1\right)^{1 / 2}}
$$

with $y_{g}:=-\frac{1}{2} \ln \left[2 g^{-1}(1-\sqrt{1-g})-1\right]$ for the conductance $g$ in the standard symmetry class (4.2).

\section{Crossover regime}

As was already the case in Sec. IV. B 3, we could not solve the Fokker-Planck equation (4.17) exactly in the crossover regime. We thus try the separation-of-variable ansatz

$$
\mathcal{Q}(\varrho, \phi ; t)=\mathcal{Q}(\varrho ; t) \Phi(\phi) .
$$

We are again assuming that the probability distribution $\Phi$ is $t$ independent and we made the specific choice for $\Phi$ given by Eq. (4.9). Insertion of Eq. (4.27) into the Fokker-Planck equation (4.17) followed by an integration over the phase $\phi$ yields

$$
\begin{aligned}
\frac{\partial \mathcal{Q}}{\partial t}= & -2 \zeta \alpha \mathcal{Q}+(2 \varrho-1)(1-2 \zeta \alpha) \frac{\partial \mathcal{Q}}{\partial \varrho} \\
& +\varrho(\varrho-1)(1-\zeta \alpha) \frac{\partial^{2} \mathcal{Q}}{\partial \varrho^{2}}
\end{aligned}
$$

with the initial condition

$$
\mathcal{Q}(\varrho ; t=0)=\delta(\varrho-1) .
$$

The normalized solution to Eq. (4.28) has the integral representation
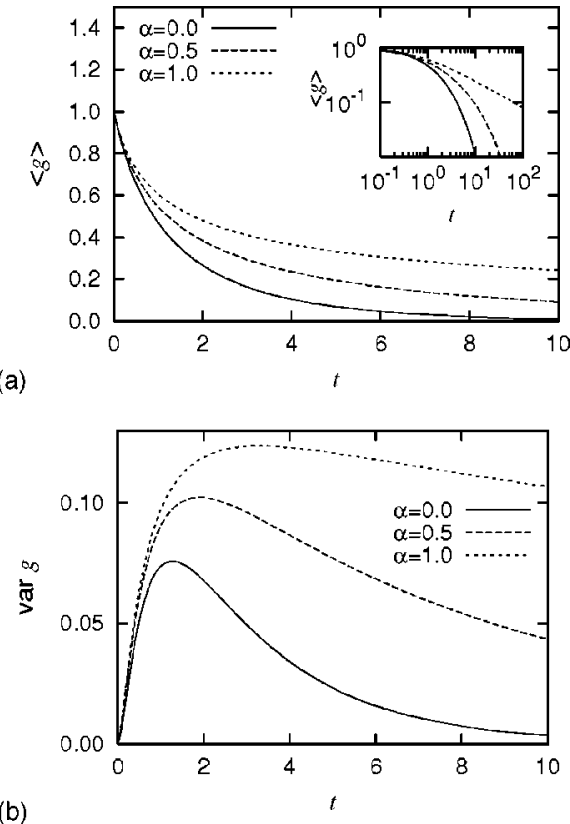

FIG. 4. The mean (a) and variance (b) of the conductance computed from the probability distribution (4.3) as a function of $t$ $=L / \ell$ for $\alpha=0,0.5,1$ and $\zeta=-1$.

$$
\begin{aligned}
\mathcal{Q}(\varrho ; t)= & \sin \left(\frac{-\pi \zeta \alpha}{1-\zeta \alpha}\right) \frac{\varrho^{\zeta \alpha /(1-\zeta \alpha)}}{\sqrt{4 \pi^{3} t^{\prime 3}}} \exp \left[-\frac{t^{\prime}(1+\zeta \alpha)^{2}}{4(1-\zeta \alpha)^{2}}\right] \\
& \times \int_{0}^{1} d u \frac{\left(\frac{1}{u}-1\right)^{\zeta \alpha /(1-\zeta \alpha)}}{[u(1-u)(\varrho-u)]^{1 / 2}} \\
& \times \int_{0}^{1} d v \frac{e^{-D^{2}(u, v ; \varrho) / 4 t^{\prime}}}{v^{1 / 2}(1-v)^{1 /(1-\zeta \alpha)}} D(u, v ; \varrho)
\end{aligned}
$$

with the auxiliary function and variable

$$
D(u, v ; \varrho):=\ln \left[\frac{\varrho-u}{v u(1-u)}\right], \quad t^{\prime}:=(1-\zeta \alpha) t
$$

As it should be, we recover Eq. (4.20) when $\zeta \alpha=-1$ and Eq. (4.24) when $\alpha=0$. The approximate probability distribution $\mathcal{G}$ for the conductance $g$ in the crossover regime is obtained from Eq. (4.29) through

$$
\mathcal{G}(g ; t)=g^{-2} \mathcal{Q}(\varrho ; t), \quad \varrho(g)=g^{-1} .
$$

The dependence on $t$ for the mean and variance of the conductance in the crossover regime computed from Eq. (4.30) are depicted in Fig. 4. In the chiral symmetry class $\zeta=-1$, $\alpha=+1$, the mean and variance decay like $t^{-1 / 2}$. For $\alpha=0.5$ and $\alpha=0$, the mean and variance decay exponentially with $t$.

\section{VALIDITY OF THE SEPARATION ANSATZ FOR THE CROSSOVER REGIME}

We have constructed an approximate solution to the Fokker-Planck equations (3.7) and (4.17) in the crossover 
regime (4.3) by assuming the separation of the squared magnitude $R$ from the phase $\phi$ of the reflection coefficient $r$. In this section we shall investigate the accuracy of this approximation to the crossover regime (4.3) for very large values of the length $L$ of the disordered quantum wire, i.e., $L$ much larger than the localization length $\xi$, which will be defined below. To this end, we shall compare the cumulants of the radial coordinate $x$ computed from the separation-of-variable ansatz with the same cumulants computed from a largedeviation ansatz introduced by Schomerus and Titov. ${ }^{25,26}$

\section{A. Large $t$ limit in the crossover regime with the separation-of-variable ansatz}

We begin from Eq. (3.15) without absorption and in the crossover regime. We seek the asymptotic behavior when $t$ $\rightarrow \infty$ of the solutions to Eq. (3.15) in the crossover regime. We try the separation-of-variable ansatz

$$
W(x, \phi ; t)=\mathcal{X}(x ; t) \Phi(\phi),
$$

where $\Phi$ is the stationary and normalized solution (4.9). Insertion of Eq. (5.1) into Eq. (3.15) with subsequent integration over $\phi$ yields

$$
\frac{\partial \mathcal{X}}{\partial t}=-\frac{1}{2}(1+\zeta \alpha) \frac{\partial \mathcal{X}}{\partial x}+\frac{1}{4}(1-\zeta \alpha) \frac{\partial^{2} \mathcal{X}}{\partial x^{2}} .
$$

Equation (5.2a) is nothing but the diffusion equation with a constant drift proportional to the amount of chiral symmetry breaking $(1+\zeta \alpha)$, i.e., it is solved by

$$
\mathcal{X}(x ; t)=\frac{\exp \left\{-[x-(1+\zeta \alpha) t / 2]^{2} /[(1-\zeta \alpha) t]\right\}}{\sqrt{\pi(1-\zeta \alpha) t}}
$$

normalized to the real line $x \in \mathbb{R}$. In the approximation (5.2b), the only nonvanishing cumulants are the first and second cumulants,

$$
\begin{gathered}
\langle x\rangle_{c}:=\langle x\rangle=\frac{1+\zeta \alpha}{2} t, \\
\left\langle x^{2}\right\rangle_{c}:=\left\langle\left(x-\frac{1+\zeta \alpha}{2} t\right)^{2}\right\rangle=\frac{1-\zeta \alpha}{2} t .
\end{gathered}
$$

Provided $\zeta \alpha \neq-1$ (i.e., away from the chiral symmetry class), the random variable $x$ becomes self-averaging in the thermodynamic limit. It then makes sense to identify the localization length $\xi$ through

$$
\langle x\rangle_{c}=\frac{\ell}{\xi} t, \quad t \rightarrow \infty,
$$

which, together with Eq. (5.3a), gives

$$
\xi=\frac{2 \ell}{1+\zeta \alpha} \text {. }
$$

In the standard symmetry class (4.2) $\alpha=0$ and the localization length is twice the mean-free path $\xi=2 \times \ell$ as is well known. ${ }^{52}$ In the chiral symmetry class (4.1) $\zeta \alpha=-1$ and the localization length defined by Eq. (5.4) is not a finite number

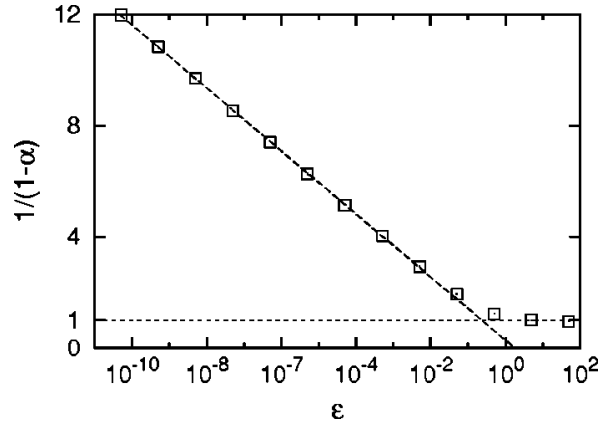

FIG. 5. Numerical evaluation of $1 /(1-\alpha)$ as a function of $\varepsilon$ in the crossover regime (4.3). The integral $\alpha$ defined in Eq. (4.15b) is evaluated numerically for the stationary distribution of the phase (4.9). The line representing $-(1 / 2) \log |\varepsilon|$ is a guide to the eyes.

anymore but a random variable as the right-hand side of Eq. (5.3a) vanishes whereas the right-hand side of Eq. (5.3b) remains finite. For the crossover regime (4.3), the constant $\alpha$ implicitly depends on $\varepsilon$. The asymptotic behavior of $\alpha$ for small $\varepsilon>0$ can be evaluated as, with the probability distribution (4.9),

$$
\begin{aligned}
\frac{1}{1-\alpha} & =\frac{\int_{-\infty}^{+\infty} d x\left(x^{2}+1\right)^{-1 / 2} \int_{-\infty}^{x} d y\left(y^{2}+1\right)^{-1 / 2} e^{\varepsilon(y-x) / 2}}{2 \int_{-\infty}^{+\infty} d x\left(x^{2}+1\right)^{-3 / 2} \int_{-\infty}^{x} d y\left(y^{2}+1\right)^{-1 / 2} e^{\varepsilon(y-x) / 2}} \\
& \sim-\frac{1}{2} \ln \varepsilon,
\end{aligned}
$$

since a logarithmic divergence occurs both in the $x$ and $y$ integrals in the numerator as we take $\varepsilon \rightarrow 0$, while it occurs only in the $y$ integral in the denominator around $y=-\infty$. It is then natural to identify

$$
\xi=\frac{2 \ell}{1+\zeta \alpha} \quad(\text { at } \zeta=-1 \quad \text { and } \text { for } \varepsilon \ll 1)
$$

with the typical localization length ${ }^{5,6}(\sim|\ln | \varepsilon|| \times \ell)$ when approaching the chiral symmetry class (4.1) through the crossover regime (4.3). We have also computed the dependence of $\alpha$ on $\varepsilon$ by evaluating Eq. (5.6) numerically. In Fig. 5, 1/(1 $-\alpha$ ) is plotted as a function of $\varepsilon$ in the crossover regime (4.3). We observe that $1 /(1-\alpha)$ asymptotically behaves as $-(1 / 2) \ln |\varepsilon|$ in the vicinity $(\varepsilon<0.1)$ of the chiral symmetry class (4.1a) and (4.1b).

\section{B. Large $t$ limit in the crossover regime with the large-deviation ansatz}

Schomerus and Titov, ${ }^{25,26}$ devised a systematic method to compute all the cumulants of the conductance and the LDOS to leading order in $\xi / L(\ll 1)$. In practice, they carried out explicitly the computation of the first four cumulants.

To draw a connection to their work we note that whereas the parametrization $x=\operatorname{arctanh} \sqrt{R}$ of the magnitude of the reflection coefficient is the natural one from a geometrical point of view according to Eqs. (2.9), (2.12), and (2.13), it is 


$$
1 \ll u:=2 x \sim-\ln g \sim-\ln \nu
$$

that behaves statistically as the logarithm of the conductance $g$ (or the logarithm of the LDOS $\nu$ according to the Borland conjecture, see Ref. 53) in the regime defined by the condition

$$
0 \leqslant g \ll 1 .
$$

We denote by $U(u, \phi ; L)$ the joint probability distribution of $u$ and $\phi$, and introduce $s \equiv 2 L / \ell$. We infer from Eq. (3.15) that $U(u, \phi ; L)$ obeys the Fokker-Planck equation

$$
\begin{aligned}
\frac{\partial U}{\partial s}= & \mathcal{L}_{\phi} U+\left(-\frac{1}{2}+\frac{3}{2} \zeta \cos 2 \phi\right) \frac{\partial U}{\partial u}+\frac{1}{2}(1-\zeta \cos 2 \phi) \frac{\partial^{2} U}{\partial u^{2}} \\
& +\zeta \sin (2 \phi) \frac{\partial^{2} U}{\partial u \partial \phi},
\end{aligned}
$$

where

$$
\begin{aligned}
\mathcal{L}_{\phi}:= & -\zeta \cos 2 \phi-\left(\frac{\varepsilon}{2}+\frac{3}{2} \zeta \sin 2 \phi\right) \frac{\partial}{\partial \phi} \\
& +\left(\zeta_{0}+\frac{1}{2}+\frac{\zeta}{2} \cos 2 \phi\right) \frac{\partial^{2}}{\partial \phi^{2}} \\
= & -\frac{\varepsilon}{2} \frac{\partial}{\partial \phi}+\zeta_{0} \frac{\partial^{2}}{\partial \phi^{2}}+\frac{g_{1}}{g_{+}}\left(\frac{\partial}{\partial \phi} \cos \phi\right)^{2}+\frac{g_{2}}{g_{+}}\left(\frac{\partial}{\partial \phi} \sin \phi\right)^{2}
\end{aligned}
$$

in the large $u$ limit and in the absence of absorption.

The fact that $\mathcal{L}_{\phi}$ can be written as a total derivative plays an essential role when solving, for any given purely imaginary $z$ and any given positive integer $k$, the eigenvalue equation

$$
\begin{aligned}
\mu_{k} f_{k}= & {\left[\mathcal{L}_{\phi}-z\left(-\frac{1}{2}+\frac{3}{2} \zeta \cos 2 \phi+\zeta \sin (2 \phi) \frac{\partial}{\partial \phi}\right)\right.} \\
& \left.+\frac{z^{2}}{2}(1-\zeta \cos 2 \phi)\right] f_{k}
\end{aligned}
$$

for the eigenvalue $\mu_{k}(z)$ and the normalized eigenfunction $f_{k}(z, \phi)$ of $\phi$ with periodicity $\pi$ that results from insertion of the large-deviation ansatz

$$
U(u, \phi ; L)=\int_{-i \infty}^{+i \infty} \frac{d z}{2 \pi i} \sum_{k=0}^{+\infty} e^{\mu_{k}(z) s-z u} f_{k}(z, \phi)
$$

into Eq. (5.9).

For large $u \gg 1$, the integration over $z$ is dominated in Eq. (5.10b) by the region of size $\sim 1 / u$ close to the origin $z=0$. Assume that for a nonvanishing $z$ of order $1 / u$, all eigenvalues $\mu_{k}(z)$ are real valued, of descending order, and that the largest eigenvalue $\mu_{k=0}(z)$ is separated from $\mu_{k=1}(z)$ by a finite gap. Consequently, truncation of the summation over $k$ to the single term $k=0$ on the right-hand side of Eq. (5.10b) produces an exponentially small error

$$
U(u, \phi ; L)=\int_{-i \infty}^{+i \infty} \frac{d z}{2 \pi i}\left[e^{\mu_{0}(z) s-z u} f_{0}(z, \phi)+\mathcal{O}\left(e^{-\left|\mu_{0}(z)-\mu_{1}(z)\right| s}\right)\right] .
$$

Next, assume the expansions

$$
\mu_{0}(z)=\sum_{n=1}^{+\infty} \mu_{0}^{(n)} z^{n}, \quad f_{0}(z, \phi)=\sum_{n=0}^{+\infty} f_{0}^{(n)}(\phi) z^{n},
$$

where the expansion coefficient $\mu_{0}^{(n)}$ encodes the $n$th cumulant of $u$,

$$
\left\langle u^{n}\right\rangle_{c}=n ! \mu_{0}^{(n)} s+\mathcal{O}\left(s^{0}\right) .
$$

Insertion of the expansion (5.12) into the Eq. (5.10a) for $k$ $=0$ can be solved iteratively for $n=0,1, \ldots$. For $n=0$, Eq. (5.10a) reduces to

$$
0=\mathcal{L}_{\phi} f_{0}^{(0)}
$$

whose normalized solution is nothing but the stationary and normalized solution to Eq. (4.4b). For $n=1$, Eq. (5.10a) reduces to

$$
\mu_{0}^{(1)} f_{0}^{(0)}=\mathcal{L}_{\phi} f_{0}^{(1)}+\left(\frac{1}{2}-\frac{3}{2} \zeta \cos (2 \phi)-\zeta \sin (2 \phi) \partial_{\phi}\right) f_{0}^{(0)} .
$$

The expansion coefficient $\mu_{0}^{(1)}$ is obtained from integrating Eq. (5.15) over $\phi$ as a result of $\mathcal{L}_{\phi}$ being a total derivative,

$$
\mu_{0}^{(1)}=\frac{1}{2}\left(1+\zeta \alpha_{0}^{(0)}\right),
$$

where

$$
\alpha_{0}^{(0)}:=\int_{0}^{2 \pi} d \phi \cos (2 \phi) f_{0}^{(0)}(\phi) .
$$

After comparison of Eqs. (5.13) and (5.16), on the one hand, with Eqs. (5.3a) and (4.15b), on the other hand, we conclude that the first cumulant of the logarithm of the conductance is computed exactly to leading order in $\xi / L$ within the separation-of-variable ansatz for the crossover regime (4.3). As a corollary, the crossover (5.5) for the typical localization length is given exactly by the separation-of-variable ansatz. Carrying on the iteration to compute the expansion coefficients $\mu_{0}^{(n)}$, however, we find that the separation-of-variable ansatz breaks down for $n=1,2, \ldots$ as it does not agree anymore with the large-deviation ansatz from Schomerus and Titov in Refs. 25 and 26.

\section{PROBABILITY DISTRIBUTION OF THE LDOS}

In this section, the probability distributions $P(\nu ; L)$ and $P(\nu ; \omega)$ of the LDOS $\nu$ in the geometries of Figs. 6(a) and 6 (b), respectively, are calculated based on the probability distribution function of the reflection coefficient $r$ $=\sqrt{R} \exp (i \phi)$ obtained in Sec. IV.

\section{A. Single-particle Green function and reflection coefficient}

For any given realization of the disorder potentials $v_{0,1,2}$, the $\operatorname{LDOS} \nu(y)$ is defined by 54 
(a)

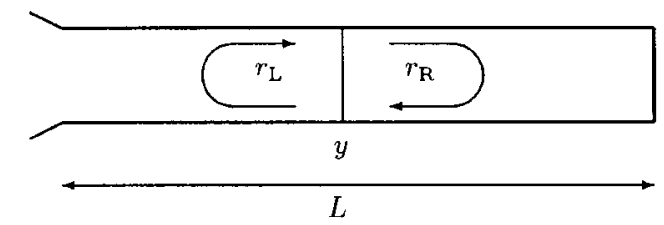

(b)

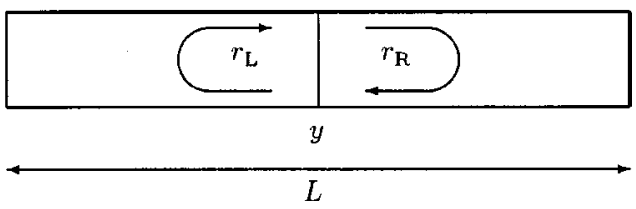

FIG. 6. (a) Disordered quantum wire of length $L$ closed at the right end and connected to a perfect lead at the left end. Energy levels within the wire of length $L$ are broadened beyond the mean level spacing by the coupling to the perfect lead. (b) Disordered quantum wire of length $L$ closed at both ends. Energy levels within the wire are broadened beyond the mean level spacing by the absorption $\operatorname{Im} E=\mathcal{E}^{\prime \prime}>0$.

$$
\nu(y):=-\frac{1}{\pi} \operatorname{Im} \operatorname{tr} G(y, y),
$$

where the matrix elements $G(y, z)$ in real space of the Green function are obtained from solving

$$
\begin{gathered}
{\left[E+i \eta+\sigma_{3} i \frac{d}{d y}+\sum_{\mu=0}^{2} \sigma_{\mu} v_{\mu}(y)\right] G(y, z)=\delta(y-z) \sigma_{0},} \\
E=\mathcal{E}^{\prime}+i \mathcal{E}^{\prime \prime}, \quad \mathcal{E}^{\prime \prime} \geqslant 0,
\end{gathered}
$$

$(\eta$ : $\quad$ positive infinitesimal)

with the boundary conditions associated to the geometries in Fig. 6. As long as $y$ is far away from the ends of the disordered quantum wire, say in the middle, the statistical properties of $\nu(y)$ should be independent of $y$. However, the statistical properties of $\nu(y)$ depend strongly on the boundary conditions at the ends of the disordered quantum wire. ${ }^{19,21}$ For completeness, we will understand, under the chiral limit of the LDOS (6.1), the property that the Green function (6.2) anticommutes with $\sigma_{1}$, i.e.,

$$
E=g_{0}=g_{1}=0,
$$

whereas we will understand, under the standard limit of the LDOS (6.1), the conditions on the Green function (6.2) that

$$
g_{1}=g_{2}
$$

or

$$
g_{0} \gg g_{1}+g_{2}
$$

or

$$
E \gg g_{1}+g_{2} .
$$

Schomerus et al. $^{21}$ expressed the probability distribution of the LDOS in terms of the probability distributions of the reflection coefficient $r_{\mathrm{L}}\left(r_{\mathrm{R}}\right)$ for outgoing plane waves re- flected from the region to the left (right) of $y$ for the continuous nonrelativistic Schrödinger equation in the standard symmetry class (6.4). Here we extend their analysis to the continuous relativistic Hamiltonian (2.1a). A crucial observation in Ref. 21 is that, for weak disorder, the Green function can be expanded in terms of free scattering states within a small interval of order of the mean-free path $\ell$ since one can assume that there are no impurities in this interval. The solution to Eq. (6.2) for $y$ and $z$ belonging to such an impurityfree interval can be written as a linear combination of $e^{i k(+y+z)} \chi_{+} \chi_{-}^{T}, e^{i k(+y-z)} \chi_{+} \chi_{+}^{T}, e^{i k(-y+z)} \chi_{-} \chi_{-}^{T}, e^{i k(-y-z)} \chi_{-} \chi_{+}^{T}$, except for the discontinuity at $y=z$,

$$
\begin{aligned}
G(y, z)= & \frac{\left(e^{-i k y} \chi_{-}+r_{\mathrm{L}} e^{+i k y} \chi_{+}\right)\left(e^{+i k z} \chi_{-}+r_{\mathrm{R}} e^{-i k z} \chi_{+}\right)^{T}}{i \hbar v_{\mathrm{F}}\left(1-r_{\mathrm{L}} r_{\mathrm{R}}\right)} \Theta(z-y) \\
& +\frac{\left(e^{+i k y} \chi_{+}+r_{\mathrm{R}} e^{-i k y} \chi_{-}\right)\left(e^{-i k z} \chi_{+}+r_{\mathrm{L}} e^{+i k z} \chi_{-}\right)^{T}}{i \hbar v_{\mathrm{F}}\left(1-r_{\mathrm{L}} r_{\mathrm{R}}\right)} \\
& \times \Theta(y-z),
\end{aligned}
$$

where $\chi_{+}:=(1,0)^{T}, \chi_{-}:=(0,1)^{T}, \Theta(y)$ is the Heaviside function that vanishes when $y<0$ and equals one when $y>0$, and we have momentarily reinstated the Fermi velocity $v_{\mathrm{F}}$ and $\hbar$ in the relativistic dispersion relation $\operatorname{Re} E \equiv \mathcal{E}^{\prime}=\hbar v_{\mathrm{F}} k$. The relative amplitudes among $e^{i k( \pm y \pm z)} \chi_{ \pm} \chi_{\mp}^{T}$ as well as the factor $\left(1-r_{\mathrm{L}} r_{\mathrm{R}}\right)^{-1}$ are determined by taking into account multiple scattering from the left and right boundaries of an interval free of impurities.

Combining Eqs. (6.2) and (6.5) reproduces in the relativistic limit the mesoscopic relation

$$
\nu(y)=\frac{1}{\pi \hbar v_{\mathrm{F}}} \operatorname{Re}\left(\frac{1+r_{\mathrm{R}} r_{\mathrm{L}}}{1-r_{\mathrm{R}} r_{\mathrm{L}}}\right)
$$

between the LDOS at $y$ and the reflection coefficients $r_{\mathrm{L}}$ and $r_{\mathrm{R}}$ from the disordered region to the left and right of $y$, respectively, derived by Schomerus et al. ${ }^{21}$ for the nonrelativistic continuous Schrödinger equation. In the nonrelativistic case, the diagonal matrix element of the Green function in real space gives the microscopic local density of states. The microscopic local density of states exhibits $2 k_{\mathrm{F}}$ oscillations. ${ }^{21}$ The LDOS (6.6) is obtained after smearing out these oscillations. The effect of the relativistic approximation (2.1a) is to remove the $2 k_{\mathrm{F}}$ oscillations from the outset. Henceforth let us measure the $\operatorname{LDOS} \nu(y)$ in units of $1 /\left(\pi \hbar v_{\mathrm{F}}\right)$, the DOS of a clean wire.

The probability distribution function $P\left(\nu ; L_{\mathrm{L}}, L_{\mathrm{R}}, \omega\right)$ of the LDOS $\nu(y)$ defined by Eq. (6.6) is now simply given by

$$
\begin{aligned}
P\left(\nu ; L_{\mathrm{L}}, L_{\mathrm{R}}, \omega\right) & \\
= & \int_{0}^{1} d R_{\mathrm{L}} \int_{0}^{1} d R_{\mathrm{R}} \int_{0}^{2 \pi} d \phi_{\mathrm{L}} \int_{0}^{2 \pi} d \phi_{\mathrm{R}} \\
& \times P_{\mathrm{L}}\left(R_{\mathrm{L}}, \phi_{\mathrm{L}} ; L_{\mathrm{L}}, \omega\right) P_{\mathrm{R}}\left(R_{\mathrm{R}}, \phi_{\mathrm{R}} ; L_{\mathrm{R}}, \omega\right) \\
& \times \delta\left(\nu-\frac{1-R_{\mathrm{L}} R_{\mathrm{R}}}{1+R_{\mathrm{L}} R_{\mathrm{R}}-2 \sqrt{R_{\mathrm{L}} R_{\mathrm{R}}} \cos \left(\phi_{\mathrm{L}}+\phi_{R}\right)}\right),
\end{aligned}
$$

where $L_{\mathrm{L}}\left(L_{\mathrm{R}}\right)$ is the length of the segment of the disordered 
quantum wire to the left (right) of the point $y$ where the LDOS is measured. The total length of the disordered quantum wire is evidently given by (see Fig. 6)

$$
L=L_{\mathrm{L}}+L_{\mathrm{R}} \text {. }
$$

The dependence of the probability distribution for the LDOS on the boundary conditions enters through the dependence of the probability distributions $P_{\mathrm{L}}\left(R_{\mathrm{L}}, \phi_{\mathrm{L}} ; L_{\mathrm{L}}, \omega\right)$ and $P_{\mathrm{R}}\left(R_{\mathrm{R}}, \phi_{\mathrm{R}} ; L_{\mathrm{R}}, \omega\right)$ on the boundary conditions corresponding to the geometries of Fig. 1. ${ }^{19,21}$ This dependence on boundary conditions is implied by the dependence on $L_{\mathrm{L}}, \omega$ or $L_{\mathrm{R}}, \omega$ of the probability distribution of the two reflection coefficients $r_{\mathrm{L}}$ and $r_{\mathrm{R}}$, respectively. When a disordered quantum wire of finite length is closed, one must broaden the single-particle energy levels beyond the mean level spacing to make sense of the energy dependence of the mesoscopic LDOS. This is achieved here by introducing a finite absorption $\omega=\operatorname{Im} E / g_{+}>0$, in which case the dependence on $L_{\mathrm{L}}$ $\gg \ell$ or $L_{R} \gg \ell$ becomes immaterial. The absorption $\omega$ $=\operatorname{Im} E / g_{+}>0$ is switched off when a disordered quantum wire of finite length $L$ is connected to a perfect lead. We consider first the geometry depicted in Fig. 6(a) and then the geometry depicted in Fig. 6(b).

\section{B. Semiopen wire}

To calculate the probability distribution of the LDOS in a disordered quantum wire connected to a reservoir on the left and closed on the right, we need (i) the joint probability distribution $P_{\mathrm{L}}\left(R_{\mathrm{L}}, \phi_{\mathrm{L}} ; L_{\mathrm{L}}\right)$ for the square $R_{\mathrm{L}}$ of the magnitude of the reflection coefficient $r_{\mathrm{L}}$ and its phase $\phi_{\mathrm{L}}$ on the segment of length $L_{\mathrm{L}}$ to the left of the point at which the LDOS is measured and (ii) the probability distribution $\Phi_{R}\left(\phi_{R} ; L_{R}\right)$ of the phase $\phi_{R}$ of the reflection coefficient $r_{R}$ on the segment of length $L_{\mathrm{R}}$ to the right of the point at which the LDOS is measured. The calculation of $\Phi_{R}\left(\phi_{R} ; L_{R}\right)$ is outlined in Sec. IV A. The calculation of $P_{\mathrm{L}}\left(R_{\mathrm{L}}, \phi_{\mathrm{L}} ; L_{\mathrm{L}}\right)$ is outlined in Sec. IV C.

\section{Chiral and standard symmetry classes}

The probability distribution of the LDOS in the chiral symmetry class (6.3) is (see the Appendix)

$$
P\left(\nu ; L_{\mathrm{L}}\right)=\frac{1}{\sqrt{8 \pi L_{\mathrm{L}} / \ell}} \frac{1}{\nu} \exp \left(-\frac{\ell}{8 L_{\mathrm{L}}} \ln ^{2} \nu\right) .
$$

This is the probability distribution of a log-normal distributed random variable with

$$
\begin{gathered}
\langle\ln \nu\rangle_{L_{\mathrm{L}}}:=\int_{0}^{\infty} d \nu P\left(\nu ; L_{\mathrm{L}}\right) \ln \nu=0, \\
\left\langle(\ln \nu)^{2}\right\rangle_{L_{\mathrm{L}}}:=\int_{0}^{\infty} d \nu P\left(\nu ; L_{\mathrm{L}}\right)(\ln \nu)^{2}=\frac{4 L_{\mathrm{L}}}{\ell} .
\end{gathered}
$$

The average LDOS is

$$
\langle\nu\rangle_{L_{\mathrm{L}}}=e^{2 L_{\mathrm{L}} / \ell},
$$

which grows exponentially with $L_{\mathrm{L}}$. For comparison, the probability distribution of the LDOS in the standard symme-
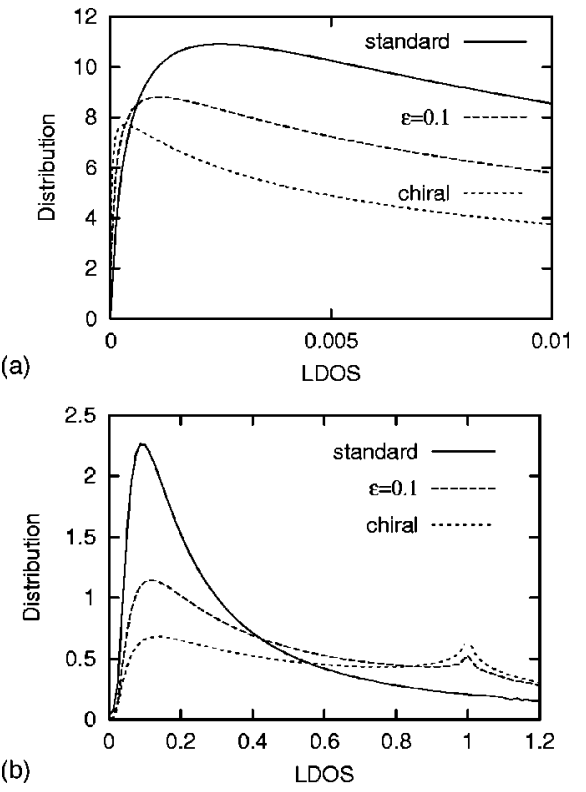

FIG. 7. (a) Three traces for the probability distribution of the LDOS are plotted with $\varepsilon=0,0.1,+\infty$ (from the bottom up at $\nu$ $=0.01)$ so as to interpolate between the chiral and standard symmetry classes (6.3) and (6.4), respectively. Each trace is obtained from numerical integration of Eq. (6.7) for the semiopen geometry of Fig. 6(a) with $L_{\mathrm{L}} / \ell=2$. (b) Three traces for the probability distribution of the LDOS are plotted with $\varepsilon=0,0.1,+\infty$ (from the bottom up at $\nu=0.1$ ) so as to interpolate between the chiral and standard symmetry classes (6.3) and (6.4), respectively. Each trace is obtained from numerical integration of Eq. (6.7) for the closed geometry of Fig. 6(b) with $\omega=1 / 12$. We have verified that numerical integration of Eq. (6.7) agrees with a representation of Eq. (6.7) in terms of elementary functions when possible.

try class (6.4), which was obtained in Refs. 19 and 21, is also log-normal,

$$
P\left(\nu ; L_{\mathrm{L}}\right)=\frac{1}{\sqrt{4 \pi L_{\mathrm{L}} / \ell}} \frac{1}{\nu} \exp \left[-\frac{\ell}{4 L_{L}}\left(\ln \nu+\frac{L_{\mathrm{L}}}{\ell}\right)^{2}\right],
$$

$$
\langle\ln \nu\rangle_{L_{\mathrm{L}}}=-\frac{L_{\mathrm{L}}}{\ell}
$$

$$
\left\langle\left(\ln \nu+\frac{L_{\mathrm{L}}}{\ell}\right)^{2}\right\rangle_{L_{L}}=\frac{2 L_{\mathrm{L}}}{\ell}
$$

Incidentally, the average LDOS is not affected by the disorder in the standard symmetry class, $\langle\nu\rangle_{\mathrm{L}_{\mathrm{L}}}=1$. The probability distributions for the LDOS in the chiral and standard symmetry classes are depicted in Fig. 7(a). In the thermodynamic limit $L_{\mathrm{L}} \rightarrow \infty$, the logarithm of the LDOS is self-averaging in the standard symmetry class whereas this is not the case in the chiral symmetry class. This difference could have been anticipated from the identification $\ln \nu \sim-2 x$, valid when $L_{\mathrm{L}} / \ell \gg 1$, with the radial coordinate $x$ that obeys the FokkerPlanck equations (2.12) and (2.13), respectively. 


\section{Separation ansatz for the crossover regime}

Numerical evaluation of the integrals on the right-hand side of Eq. (6.7) is depicted in Fig. 7(a) for the crossover regime (4.3) within our approximation which is encoded by the separation-of-variable ansatz (4.27)-(4.29), and (4.9).

\section{Closed wire}

To calculate the probability distribution of the LDOS in a disordered quantum closed at both ends with a finite absorption $\omega$, we need the joint probability distributions $P_{\mathrm{L}}\left(R_{\mathrm{L}}, \phi_{\mathrm{L}} ; L_{\mathrm{L}}, \omega\right)$ and $P_{\mathrm{R}}\left(R_{\mathrm{R}}, \phi_{\mathrm{R}} ; L_{\mathrm{R}}, \omega\right)$. We can safely ignore the dependence on $L_{\mathrm{L}, \mathrm{R}}$ in the regime $L_{\mathrm{R} / \mathrm{L}} \gg \ell$ as is done in Sec. IV B.

\section{Chiral and standard symmetry classes}

The probability distribution of the LDOS in the chiral symmetry class (6.3) is ${ }^{55}$ (see the Appendix)

$$
P(\nu ; \omega)=\left[\frac{1}{K_{0}(\omega / 2)}\right]^{2} \frac{1}{\nu} \exp \left[-\frac{\omega\left(1+\nu^{2}\right)}{2 \nu}\right] K_{0}\left(\frac{\omega\left|1-\nu^{2}\right|}{2 \nu}\right),
$$

where $K_{n}(x), n=0,1, \ldots$ are the modified Bessel functions and has the asymptotics

$$
P(\nu ; \omega) \propto\left\{\begin{array}{l}
\nu^{-1 / 2} \exp (-\omega / \nu), \quad \text { for } \quad \nu \ll \omega / 2, \\
-\ln (\omega|1-\nu|), \quad \text { for } \quad|1-\nu| \ll \frac{1}{\omega}, \\
\nu^{-3 / 2} \exp (-\omega \nu), \quad \text { for } \quad \nu \gg 2 / \omega .
\end{array}\right.
$$

For comparison, the probability distribution of the LDOS in the standard symmetry class (6.4), which was obtained in Refs. 19 and 21,

$$
\begin{aligned}
P(\nu ; \omega)= & \frac{\omega^{2}}{\sqrt{2} \pi \nu^{3 / 2}} \int_{\left(1+\nu^{2}\right) /(2 \nu)}^{\infty} d u \frac{\exp [-\omega(u-1)]}{\sqrt{u-\left(1+\nu^{2}\right) /(2 \nu)}} \\
& \times\left[u K_{0}\left(\omega \sqrt{u^{2}-1}\right)+\sqrt{u^{2}-1} K_{1}\left(\omega \sqrt{u^{2}-1}\right)\right],
\end{aligned}
$$

has the asymptotics

$$
P(\nu ; \omega) \propto \begin{cases}\nu^{-2} \exp (-\omega / \nu), & \text { for } \quad \nu \ll \omega / 2, \\ \nu^{-1 / 2} \exp (-\omega \nu), & \text { for } \quad \nu \gg 2 / \omega .\end{cases}
$$

The probability distributions for the LDOS in the chiral and standard symmetry classes are depicted in Fig. 7(b). Spectral weight from the tails $\nu \ll \omega / 2$ and $\nu \gg 2 / \omega$ of the probability distribution in the standard symmetry class is redistributed around $\nu=1$ in the chiral symmetry class. Consequently, in the limit $\omega \rightarrow 0$, the mean value of the mesoscopic LDOS in the chiral symmetry class becomes

$$
\begin{aligned}
\langle\nu\rangle_{\omega}= & \frac{1}{\left[K_{0}(\omega / 2)\right]^{2}} \int_{1}^{\infty} d \nu\left(1+\frac{1}{\nu^{2}}\right) \\
& \times \exp \left[-\frac{\omega}{2}\left(\nu+\frac{1}{\nu}\right)\right] K_{0}\left(\frac{\omega}{2}\left(\nu-\frac{1}{\nu}\right)\right) \\
\omega \rightarrow 0 & =\frac{1}{(\omega / 2)[\ln (\omega / 2)]^{2}}
\end{aligned}
$$

This result is identical to the smeared Dyson singularity of the DOS in the chiral symmetry class,

$$
\begin{aligned}
\nu_{\text {Dyson }}(\omega): & =\int_{-\infty}^{\infty} d \varepsilon \frac{2 \pi}{|\varepsilon||\ln | \varepsilon /\left.2\right|^{3}} \frac{1}{\pi} \frac{\omega}{\varepsilon^{2}+\omega^{2}} \\
& =\frac{1}{(\omega / 2)[\ln (\omega / 2)]^{2}} .
\end{aligned}
$$

\section{Separation ansatz for the crossover regime}

Numerical evaluation of the integrals on the right-hand side of Eq. (6.7) is depicted in Fig. 7(b) for the crossover regime (4.3) within our approximation which is encoded by the separation-of-variable ansatz (4.14), (4.15), and (4.9).

\section{CONCLUSION}

The probability distribution of the mesoscopic local density of states (LDOS) $\nu$ for a strictly one-dimensional problem of Anderson localization with chiral symmetry (the chiral symmetry class) was computed in closed form for two simply connected geometries assuming a weak disorder. As is the case when the chiral symmetry is maximally broken (the standard symmetry class), the probability distribution of $\nu$ strongly depends on boundary conditions. In a semiopen geometry we found that the probability distribution of $\nu$ is log-normal with a vanishing mean as opposed to log-normal with a finite mean in the standard symmetry class. In a closed geometry with absorption we found that the probability distribution of $\nu$ has a double-peak structure whereby the second peak turns out to be a logarithmic singularity at $\nu$ $=1$. We verified that the smeared Dyson singularity is reproduced by the mean value of the LDOS when the absorption is sufficiently small. Furthermore, we found one exact and proposed two approximate solutions to the functional renormalization group equation obeyed by the joint probability distribution for the squared modulus and the phase of the reflection amplitude of a finite and possibly dissipative wire with semi open and open boundary conditions, respectively, from which we could extract the approximate crossover of the LDOS $\nu$ and conductance $g$ between the chiral and standard symmetry classes. We could show that our approximation is exact for the first cumulant, but fails to describe higher cumulants of the logarithms of $\nu$ and $g$ in the crossover regime. 


\section{ACKNOWLEDGMENTS}

We are indebted to P. W. Brouwer for an initial collaboration on this project and for his generous feedback at later stages. We would also like to thank H. Schomerus and M. Titov for useful comments. This work was supported in part by Japan Society for the Promotion of Science (S.R.).

\section{APPENDIX: PROBABILITY DISTRIBUTION OF THE LDOS IN THE CHIRAL SYMMETRY CLASS}

In the appendix we prove Eqs. (6.9a) and (6.12a). We make use of the fact that the probability distributions for the squared modulus and phases of the reflection coefficients $r_{\mathrm{L} / \mathrm{R}}=\tanh x_{\mathrm{L} / \mathrm{R}} \exp \left(i \phi_{\mathrm{L} / \mathrm{R}}\right)$ factorize in the chiral symmetry class. We have chosen here to represent $0 \leqslant\left(R_{\mathrm{L} / \mathrm{R}}\right)^{1 / 2} \leqslant 1$ by $\tanh x_{\mathrm{L} / \mathrm{R}}$. We thus need to compute

$$
\begin{aligned}
P\left(\nu ; L_{\mathrm{L}}, L_{\mathrm{R}}, \omega\right)= & \int_{0}^{\infty} d x_{\mathrm{L}} \int_{0}^{\infty} d x_{\mathrm{R}} \int_{0}^{2 \pi} d \phi_{\mathrm{L}} \int_{0}^{2 \pi} d \phi_{\mathrm{R}} P_{\mathrm{L}}\left(x_{\mathrm{L}}, \phi_{\mathrm{L}} ; L_{\mathrm{L}}, \omega\right) P_{\mathrm{R}}\left(x_{\mathrm{R}}, \phi_{\mathrm{R}} ; L_{\mathrm{R}}, \omega\right) \\
& \times \delta\left(\nu-\frac{1-\tanh ^{2} x_{\mathrm{L}} \tanh ^{2} x_{\mathrm{R}}}{1+\tanh ^{2} x_{\mathrm{L}} \tanh ^{2} x_{\mathrm{R}}-2 \tanh x_{\mathrm{L}} \tanh x_{\mathrm{R}} \cos \left(\phi_{\mathrm{L}}+\phi_{\mathrm{R}}\right)}\right)
\end{aligned}
$$

where

$$
P_{\mathrm{L} / \mathrm{R}}\left(x_{\mathrm{L} / \mathrm{R}}, \phi_{\mathrm{L} / \mathrm{R}} ; L_{\mathrm{L} / \mathrm{R}}, \omega\right)=\mathcal{X}_{\mathrm{L} / \mathrm{R}}\left(x_{\mathrm{L} / \mathrm{R}} ; L_{\mathrm{L} / \mathrm{R}}, \omega\right) \frac{1}{2}\left[\delta\left(\phi_{\mathrm{L} / \mathrm{R}}-0\right)+\delta\left(\phi_{\mathrm{L} / \mathrm{R}}-\pi\right)\right] .
$$

Integration over the angles $\phi_{\mathrm{L} / \mathrm{R}}$ yields

$$
P\left(\nu ; L_{\mathrm{L}}, L_{\mathrm{R}}, \omega\right)=\int_{0}^{\infty} d x_{\mathrm{L}} \int_{0}^{\infty} d x_{\mathrm{R}} \mathcal{X}_{\mathrm{L}}\left(x_{\mathrm{L}} ; L_{\mathrm{L}}, \omega\right) \mathcal{X}_{\mathrm{R}}\left(x_{\mathrm{R}} ; L_{\mathrm{R}}, \omega\right) \frac{1}{2}\left[\delta\left(\nu-\frac{1-\tanh x_{\mathrm{L}} \tanh x_{\mathrm{R}}}{1+\tanh x_{\mathrm{L}} \tanh x_{\mathrm{R}}}\right)+\delta\left(\nu-\frac{1+\tanh x_{\mathrm{L}} \tanh x_{\mathrm{R}}}{1-\tanh x_{\mathrm{L}} \tanh x_{\mathrm{R}}}\right)\right] .
$$

\section{Semiopen wire}

Equation (2.13) implies that we need to evaluate Eq. (A2) with

$$
\begin{gathered}
\mathcal{X}_{\mathrm{L}}\left(x_{\mathrm{L}} ; L_{\mathrm{L}}\right)=\frac{2}{\sqrt{2 \pi L_{\mathrm{L}} / \ell}} \exp \left[-\frac{x_{\mathrm{L}}^{2}}{\left(2 L_{\mathrm{L}} / \ell\right)}\right], \\
\mathcal{X}_{\mathrm{R}}\left(x_{\mathrm{R}} ; L_{\mathrm{R}}\right)=\delta\left(x_{\mathrm{R}}-\infty\right) .
\end{gathered}
$$

It is found that

$$
P\left(\nu ; L_{\mathrm{L}}\right)=\frac{1}{2} \int_{-\infty}^{\infty} d x \mathcal{X}_{\mathrm{L}}\left(x ; L_{\mathrm{L}}\right) \delta(\nu-\exp (2 x))
$$

Integration over $x$ yields Eq. (6.9a).

\section{Closed wire}

Equation (4.11b) implies that we need to evaluate Eq. (A2) with

$$
\begin{aligned}
& \mathcal{X}_{\mathrm{L}}\left(x_{\mathrm{L}} ; \omega\right)=2 \mathcal{N}_{\omega} e^{-(\omega / 2) \cosh 2 x_{\mathrm{L}},} \\
& \mathcal{X}_{R}\left(x_{\mathrm{R}} ; \omega\right)=2 \mathcal{N}_{\omega} e^{-(\omega / 2) \cosh 2 x_{\mathrm{R}},}
\end{aligned}
$$

where $\mathcal{N}_{\omega}=\left[K_{0}(\omega / 2)\right]^{-1}$. It is found that

$$
P(\nu ; \omega)=2\left(\mathcal{N}_{\omega}\right)^{2} \int_{1}^{\infty} d p \int_{1}^{\infty} d q \frac{e^{-\omega p q} \delta(\nu-(p / q))}{\sqrt{\left(p^{2}-1\right)\left(q^{2}-1\right)}}
$$

Integration over $p$ and $q$ yields Eq. (6.12a).
${ }^{1}$ For a review see B. Kramer and A. MacKinnon, Rep. Prog. Phys. 56, 1469 (1993).

${ }^{2}$ For a review see B. Huckestein, Rev. Mod. Phys. 67, 357 (1995).

${ }^{3}$ F. J. Dyson, Phys. Rev. 92, 1331 (1953).

${ }^{4}$ G. Theodorou and M. H. Cohen, Phys. Rev. B 13, 4597 (1976).

${ }^{5}$ T. P. Eggarter and R. Riedinger, Phys. Rev. B 18, 569 (1978).

${ }^{6}$ L. Balents and M. P. A. Fisher, Phys. Rev. B 56, 12970 (1997).

${ }^{7}$ L. Fleishman and D. C. Licciardello, J. Phys. C 10, L125 (1977).

${ }^{8}$ A. D. Stone and J. D. Joannopoulos, Phys. Rev. B 24, 3592
(1981).

${ }^{9}$ M. R. Zirnbauer, J. Math. Phys. 37, 4986 (1996).

${ }^{10}$ T. Nagao and K. Slevin, J. Math. Phys. 34, 2075 (1993); J. J. M. Verbaarschot and I. Zahed, Phys. Rev. Lett. 70, 3852 (1993); S. Hikami and A. Zee, Nucl. Phys. B 408, 415 (1993); A. V. Andreev, B. D. Simons, and N. Taniguchi, ibid. 432, 487 (1994).

${ }^{11}$ Y. V. Fyodorov and A. Ossipov, Phys. Rev. Lett. 92, 084103 (2004).

${ }^{12}$ P. W. Brouwer, C. Mudry, and A. Furusaki, Phys. Rev. Lett. 84, 
2913 (2000).

${ }^{13}$ M. Titov, P. W. Brouwer, A. Furusaki, and C. Mudry, Phys. Rev. B 63, 235318 (2001).

${ }^{14}$ A. Altland and R. Merkt, Nucl. Phys. B 607, 511 (2001).

${ }^{15}$ R. Gade, Nucl. Phys. B 398, 499 (1993); R. Gade and F. Wegner, ibid. 360, 213 (1991); F. J. Wegner, Phys. Rev. B 19, 783 (1979).

${ }^{16}$ O. Motrunich, K. Damle, and D. A. Huse, Phys. Rev. B 65, 064206 (2002).

${ }^{17}$ B. Horovitz and P. Le Doussal, Phys. Rev. B 65, 125323 (2002).

${ }^{18}$ C. Mudry, S. Ryu, and A. Furusaki, Phys. Rev. B 67, 064202 (2003).

${ }^{19}$ B. L. Altshuler and V. E. Prigodin, Sov. Phys. JETP 68, 198 (1989).

${ }^{20}$ J. E. Bunder and R. H. McKenzie, Nucl. Phys. B 592, 445 (2001).

${ }^{21}$ H. Schomerus, M. Titov, P. W. Brouwer, and C. W. J. Beenakker, Phys. Rev. B 65, 121101 (2002).

${ }^{22}$ C. Mudry, P. W. Brouwer, and A. Furusaki, Phys. Rev. B 62, 8249 (2000).

${ }^{23}$ P. W. Brouwer, A. Furusaki, and C. Mudry, Phys. Rev. B 67, 014530 (2003).

${ }^{24}$ L. I. Deych, D. Zaslavsky, and A. A. Lisyansky, Phys. Rev. Lett. 81, 5390 (1998); L. I. Deych, A. A. Lisyansky, and B. L. Altshuler, ibid. 84, 2678 (2000); L. I. Deych, M. V. Erementchouk, A. A. Lisyansky, and B. L. Altshuler, ibid. 91, 096601 (2003).

${ }^{25}$ H. Schomerus and M. Titov, Phys. Rev. B 67, 100201 (2003).

${ }^{26}$ H. Schomerus and M. Titov, Eur. Phys. J. B 35, 421 (2003).

${ }^{27}$ M. Titov and H. Schomerus, Phys. Rev. Lett. 91, 176601 (2003).

${ }^{28}$ V. Dossetti-Romero, F. M. Izrailev, and A. A. Krokhin, Phys. Lett. A 320, 276 (2004).

${ }^{29}$ H. Schomerus and C. W. J. Beenakker, Phys. Rev. Lett. 84, 3927 (2000)

${ }^{30}$ O. Motrunich, K. Damle, and D. A. Huse, Phys. Rev. B 63, 224204 (2001).

${ }^{31}$ P. W. Brouwer, C. Mudry, B. D. Simons, and A. Altland, Phys. Rev. Lett. 81, 862 (1998).

${ }^{32}$ P. A. Lee, T. M. Rice, and P. W. Anderson, Phys. Rev. Lett. 31, 462 (1973)

${ }^{33}$ A. Hüffmann, J. Phys. A 23, 5733 (1990).

${ }^{34}$ P. W. Brouwer, C. Mudry, and A. Furusaki, Nucl. Phys. B 565,
$653(2000)$

${ }^{35}$ P. W. Anderson, D. J. Thouless, E. Abrahams, and D. S. Fisher, Phys. Rev. B 22, 3519 (1980).

${ }^{36}$ P. A. Mello, P. Pereyra, and N. Kumar, Ann. Phys. (N.Y.) 181, 290 (1988).

${ }^{37}$ O. N. Dorokhov, JETP Lett. 36, 318 (1982).

${ }^{38}$ H. Mathur, Phys. Rev. B 56, 15794 (1997).

${ }^{39}$ B. Shapiro, Phys. Rev. B 34, 4394 (1986).

${ }^{40}$ B. Shapiro, Philos. Mag. B 56, 1031 (1987).

${ }^{41}$ A. Cohen, Y. Roth, and B. Shapiro, Phys. Rev. B 38, 12125 (1988).

${ }^{42}$ J. Zinn-Justin, Quantum Field Theory and Critical Phenomena (Oxford University Press, New York, 1989).

${ }^{43}$ An alternative derivation of the Fokker-Planck equation (3.7a), (3.7b), and (3.7c) is the following. First, a discrete Langevin process with the discrete time step " $\delta L$ " is written down by expanding $r_{\delta L}$ in Eq. (3.2) up to second order in $v_{0,1,2}$. Second, a continuous Fokker-Planck equation for the probability distribution

$$
P(r ; L):=\left\langle\delta\left(r_{L}-r\right)\right\rangle_{\delta L}
$$

is derived from the discrete Langevin process using the methods of Appendix A3.5 of Ref. 42, say. Here, $\langle\ldots\rangle_{\delta L}$ denotes averaging over $v_{0,1,2}$ restricted to the thin slice of length $\delta L$.

${ }^{44}$ A. A. Abrikosov, Solid State Commun. 37, 997 (1981).

${ }^{45}$ V. I. Melnikov, Fiz. Tverd. Tela (S.-Peterburg) 23, 782 (1981) [Sov. Phys. Solid State 23, 444 (1981)].

${ }^{46}$ N. Kumar, Phys. Rev. B 31, 5513 (1985).

${ }^{47}$ R. Rammal and B. Doucot, J. Phys. (Paris) 48, 509 (1987).

${ }^{48}$ M. Kappus and F. Wegner, Z. Phys. B: Condens. Matter 45, 15 (1981).

${ }^{49} \mathrm{M}$. Titov (private communication).

${ }^{50}$ The crossover between the chiral and standard symmetry class induced by tuning $\zeta_{0}$ or $\zeta$ with the constraint that $\varepsilon=0$ can be studied by using the solution (4.7c).

${ }^{51}$ P. Pradhan and N. Kumar, Phys. Rev. B 50, 9644 (1994).

${ }^{52}$ For a review see C. W. J. Beenakker, Rev. Mod. Phys. 69, 731 (1997).

${ }^{53}$ R. E. Borland, Proc. R. Soc. London, Ser. A 274, 529 (1963).

${ }^{54}$ K. B. Efetov, Supersymmetry in Disorder and Chaos (Cambridge University Press, Cambridge, England, 1997).

${ }^{55} \mathrm{P}$. W. Brouwer and C. Mudry (unpublished). 